\title{
Searching for Be Stars in the Open Clusters with PTF/iPTF. I. Cluster Sample and Be Star Candidates
}

\author{
Po-Chieh Yu (俞伯傑) ${ }^{1}$ (D) , Chang-Hsien Yu (游昌憲) $)^{1}$, Chien-De Lee (李建德 $)^{1} \mathbb{1}$, Chien-Cheng Lin (林建爭 $)^{2,3}$, \\ Chih-Hao Hsia (夏志浩 $)^{4}$, Chang-Kao Chang (章展誥) ${ }^{1}$, I-Chenn Chen (陳以忱) ${ }^{1}$ (1) , Chow-Choong Ngeow (饒兆聰) ${ }^{1}{ }^{1}$, \\ Wing-Huen Ip (葉永烜 $)^{1,4,5}$, Wen-Ping Chen (陳文屏 $)^{1,6}$ (10), Russ Laher (良主嶺亞 $)^{7}$, Jason Surace ${ }^{8} \mathbb{C}$, and \\ Shrinivas R. Kulkarni ${ }^{9}$ (i) \\ ${ }^{1}$ Graduate Institute of Astronomy, National Central University, No. 300, Zhongda Road, Zhongli Dist., Taoyuan City 32001, Taiwan \\ ${ }^{2}$ Key Laboratory for Research in Galaxies and Cosmology, Shanghai Astronomical Observatory, Chinese Academy of Sciences, \\ 80 Nandan Road, Shanghai 200030, People's Republic of China \\ ${ }^{3}$ Max Planck Institute for Astronomy, Königstuhl 17, D-69117 Heidelberg, Germany \\ ${ }^{4}$ Space Science Institute, Macau University of Science and Technology, Avenida Wai Long, Taipa, Macau \\ ${ }^{5}$ Institute of Space Science, National Central University, No. 300, Zhongda Road, Zhongli Dist., Taoyuan City 32001, Taiwan \\ ${ }^{6}$ Department of Physics, National Central University, No. 300, Zhongda Road, Zhongli Dist., Taoyuan City 32001, Taiwan \\ ${ }^{7}$ Infrared Processing and Analysis Center, California Institute of Technology, Pasadena, CA 91125, USA \\ ${ }^{8}$ Spitzer Science Center, California Institute of Technology, M/S 314-6, Pasadena, CA 91125, USA \\ ${ }^{9}$ Division of Physics, Mathematics and Astronomy, California Institute of Technology, Pasadena, CA 91125, USA \\ Received 2017 June 6; revised 2017 December 26; accepted 2017 December 26; published 2018 January 29
}

\begin{abstract}
We conducted a search for Be star candidates in open clusters using $\mathrm{H} \alpha$ imaging photometry of the Palomar Transient Factory Survey to investigate some connections among Be star phenomena, cluster environments, and ages. Stellar members of clusters were identified by spatial distributions, near-infrared magnitudes and colors, and by proper motions. Among 104 open clusters, we identified $96 \mathrm{Be}$ star candidates in 32 clusters; 11 of our candidates have been reported in previous studies. We found that the clusters with age $7.5<\log (t($ year $)) \leqslant 8.5$ tend to have more Be star candidates; there is about a $40 \%$ occurrence rate within this age bin. The clusters in this age bin also tend to have a higher $\mathrm{Be}$ fraction $\mathrm{N}(\mathrm{Be}) / \mathrm{N}(\mathrm{Be}+\mathrm{B}$-type). These results suggest that the environments of young and intermediate clusters are favorable to the formation of Be phenomena. Spatial distribution of Be star candidates with different ages implies that they do not form preferentially in the central regions. Furthermore, we showed that the mid-infrared (MIR) colors of the Be star candidates are similar to known Be stars, which could be caused by free-free emission or bound-free emission. Some Be star candidates might have no circumstellar dust according to their MIR colors. Finally, among $96 \mathrm{Be}$ candidates, we discovered that one Be star candidate FSR 0904-1 exhibits long-term variability on the timescale of 2000 days with an amplitude of 0.2-0.3 mag, indicating a long timescale of disk evolution.
\end{abstract}

Key words: open clusters and associations: general - stars: emission-line, Be - stars: general - surveys

\section{Introduction}

Non-supergiant B-type stars with hydrogen emission lines, historically $\mathrm{H} \beta$ or more intense $\mathrm{H} \alpha$ emission lines, are called $\mathrm{Be}$ stars (Merrill et al. 1925; Jaschek et al. 1981). It is commonly accepted that their Balmer emission lines originated from circumstellar disks. The formation of the disks might be due to joined effects of fast rotation and of non-radial pulsations or magnetic fields (Lee et al. 1991; Porter \& Rivinius 2003; Carciofi et al. 2009). By means of fast rotation with $70 \%-80 \%$ of the break-up velocity (Townsend et al. 2004), material of the stellar surface can be removed to form the circumstellar disk. Using near-infrared spectro-interferometry with high spectral resolution $(\lambda / \Delta \lambda \sim 12,000)$, Kraus et al. (2012) studied the kinematics of two Be stars, $\beta$ Canis Minoris and $\zeta$ Tauri, and concluded that the disk is in Keplerian rotation. Their results also support the decretion disk model, e.g., the disk is replenished by the material from the star (Rivinius et al. 2013; Klement et al. 2015). Some Be stars exhibit photometric and spectroscopic variability that could be caused by stellar winds or pulsations (Rivinius et al. 2003; McSwain et al. 2009; Barnsley \& Steele 2013; Rivinius et al. 2016; Chojnowski et al. 2017; Labadie-Bartz et al. 2017; Pápics et al. 2017). Moreover, Be stars are also characterized by infrared color excess, which might be due to free-free and bound-free radiation from the ionized gas of the disks (Woolf et al. 1970; Allen 1973; Dachs \& Wamsteker 1982; Lee \& Chen 2011; Chen et al. 2016; Lee et al. 2016).

It is a mystery why only some B-type stars are Be stars. To answer this question, the essential step is to reveal the origin/ formation of the fast rotator. The first possibility is that they are natural fast rotators. Some B-types stars have been found to have a rapid rotator with an equatorial velocity of $\sim 200 \mathrm{~km} \mathrm{~s}^{-1}$ (McNally 1965; Saio et al. 2017). Another hypothesis is that Be stars could be the consequence of binary interactions. Mass gainer stars might be spun-up to fast rotators through the transfer of mass and angular momentum (McSwain \& Gies 2005). Another explanation is that they could become fast rotators during the main sequence evolution (Fabregat \& Torrejón 2000), or near the turn-off (Keller et al. 2001). These Be stars are found in young $(\sim 10 \mathrm{Myr})$ star clusters in the Milky Way (MW) galaxy (Mathew et al. 2008), Large Magellanic Cloud, and Small Magellanic Cloud (SMC; Wisniewski \& Bjorkman 2006).

Collecting a large sample of Be stars is crucial to understanding their nature. Recently, several large spectroscopic and photometric surveys have been conducted to search 
for Be stars. Using the INT Photometric $\mathrm{H} \alpha$ Survey of the Northern Galactic Plane (IPHAS; Drew et al. 2005), Raddi et al. (2015) spectroscopically confirmed 247 faint Be stars with brightness $13<r<16$ mag in the direction of the Perseus Arm. Chojnowski et al. (2015) presented 128 new Be stars with high-resolution near-infrared spectra $(\lambda / \Delta \lambda=22,500)$ from the Apache Point Observatory Galactic Evolution Experiment (APOGEE; Majewski et al. 2015). Also, Lin et al. (2015) discovered 180 Be stars identified by the Large Sky Area Multi-Object fiber Spectroscopic Telescope (LAMOST; Cui et al. 2012).

Because age information of Be stars is the key to studying their evolutions, it is necessary to collect Be stars in a sample of star clusters, as members of clusters share similar ages and environments. The enhancement of Be stars in young clusters has been found in several surveys (McSwain \& Gies 2005; Wisniewski \& Bjorkman 2006; Mathew et al. 2008; Mathew \& Subramaniam 2011; Marsh Boyer et al. 2012; Tarasov \& Malchenko 2012). McSwain \& Gies (2005) conducted an extensive survey of Be stars in 55 open clusters of the MW. They showed an increase of Be fraction with age until $100 \mathrm{Myr}$, and concluded that Be stars should be spun-up during binary interactions. Mathew et al. (2008) conducted a Be star survey with slitless spectroscopy in 207 open clusters and found that only about $20 \%$ of the clusters have Be stars. Using optical spectroscopy, Mathew \& Subramaniam (2011) investigated Be stars in 39 open clusters and found that $48 \%$ of their Be stars have $\mathrm{H} \alpha$ equivalent width (EW) in the range from -10 to $-30 \AA$. They also discovered that spectra of more than $80 \%$ of Be stars show Fe II lines. Furthermore, Martayan et al. (2009) searched for Be stars in 84 open clusters of the SMC; they showed that Be stars in the SMC are 2-4 times more abundant than in the MW. This effect is likely caused by lower metallicity of the SMC (see detailed review in Rivinius et al. 2013).

Modern surveys with wide field cameras, such as the Palomar Transient Factory (PTF; Law et al. 2009) and intermediate PTF $^{10}$ (iPTF; Kulkarni 2013), benefit not only from time saving in a comprehensive survey, but also the coverage of an entire cluster occupying a wide sky area. The data of PTF/iPTF were taken by the 48 inch Samuel Oschin Telescope located at Palomar Observatory, using an 11-CCDmosaic camera with a 7.3 square-degree field of view. The $\mathrm{PTF} / \mathrm{iPTF}$ project employed various camera filters (some have yet to be documented), including broadband filters for the $g, R$, and $i$ bands, and narrow-band filters for $\mathrm{H} \alpha 6563 \AA$ (on), and $\mathrm{H} \alpha 6630 \AA$ (off), and, more recently, for $\mathrm{H} \alpha 6720 \AA$ and $\mathrm{H} \alpha$ $6810 \AA$. The wide field of the PTF/iPTF H $\alpha$ images allow us to conduct a program to search for Be star candidates in open clusters of different ages (Yu et al. 2015, 2016). In the first of a series (Paper I), we present the cluster sample and their basic properties in Section 2. In Section 3, we describe the data and the method of identifying cluster membership and Be star candidates. We present our results and discussions in Section 4 and summarize this study in Section 5. In Paper II, we will investigate the spectroscopic properties of the $\mathrm{Be}$ star candidates. In Paper III, optical variability of a large Be star sample will be presented using current archival light curves and

\footnotetext{
${ }^{10}$ https://www.ptf.caltech.edu/iptf
}

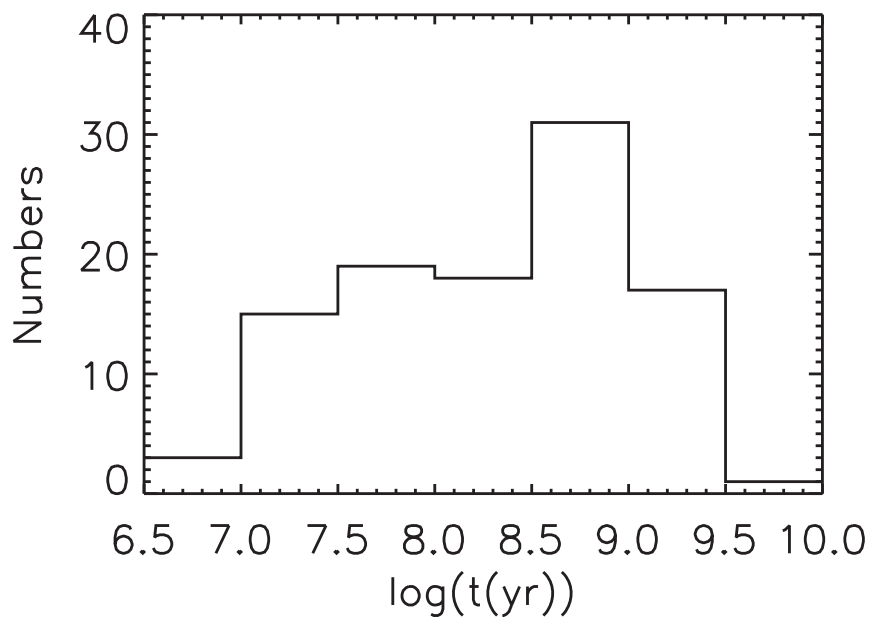

Figure 1. Age distribution of the cluster sample. Our sample covers cluster ages from a few Myr to a few Gyr.

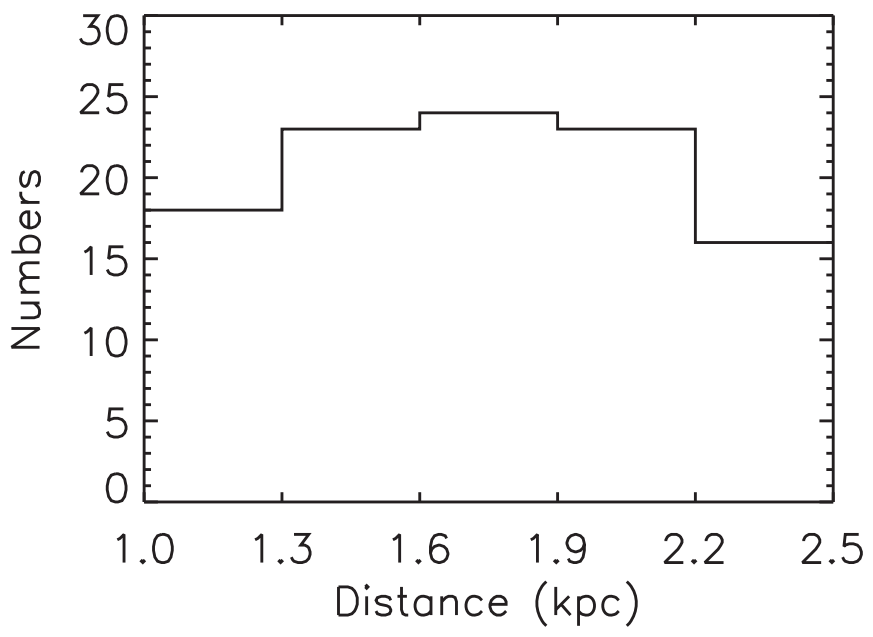

Figure 2. Distance distribution of the cluster sample. Our sample covers clusters with distance from the Sun between 1 and $2.5 \mathrm{kpc}$.

the incoming Zwicky Transient Facility survey $^{11}$ (ZTF; Kulkarni 2016).

\section{Cluster Sample}

We selected 104 clusters from Kharchenko et al. (2013) on the basis of following criteria: (1) to study the connection between $\mathrm{Be}$ star and ages, we have selected cluster ages with $6.5<\log (t($ year $)) \leqslant 10$ (Figure 1); (2) to avoid difficulties of membership identification near the Galactic center, we have selected clusters located at Galactic-longitude range $30^{\circ}<l<$ $240^{\circ}$; (3) to avoid too many bright, and to extend the sample of faint, Be stars, we constrained cluster distance from the Sun within $1 \mathrm{kpc}<D<2.5 \mathrm{kpc}$ (Figure 2). Table 1 lists the properties of the cluster sample. Notes about individual clusters are listed in Appendix. In this study, we only present clusters with more detailed studies in the Appendix.

${ }^{11}$ https://www.ptf.caltech.edu/ztf 
Table 1

Basic Properties of the Cluster Sample

\begin{tabular}{|c|c|c|c|c|c|c|c|c|c|c|c|}
\hline Name & $\begin{array}{c}\text { R.A. } \\
\text { (hh:mm:ss.ss) }\end{array}$ & $\begin{array}{c}\text { Decl. } \\
\text { (dd:mm:ss.ss) }\end{array}$ & $\begin{array}{c}\mu_{\alpha} \cos \delta \\
\left(\mathrm{mas} \mathrm{yr}^{-1}\right)\end{array}$ & $\begin{array}{c}\mu_{\delta} \\
\left(\operatorname{mas~yr}^{-1}\right)\end{array}$ & $\begin{array}{l}\text { Distance } \\
(\mathrm{kpc})\end{array}$ & $\begin{array}{c}\log \\
(t \\
\text { (year)) }\end{array}$ & $\begin{array}{l}\text { Uncert. } \\
\text { (years) }\end{array}$ & $\begin{array}{c}\text { Size } \\
(\operatorname{arcmin})\end{array}$ & $\begin{array}{l}\mathrm{N}(\mathrm{Be}) / \\
\mathrm{K}(\mathrm{Be})\end{array}$ & $\begin{array}{l}\mathrm{N}(\mathrm{Be} \\
+\mathrm{B}- \\
\text { type })\end{array}$ & References \\
\hline Mayer 1 & $00: 22: 03.36$ & $61: 44: 27.60$ & -3.87 & -3.27 & 1.430 & 7.74 & $\ldots$ & 9.8 & 0 & 23 & $\ldots$ \\
\hline Stock 20 & $00: 25: 15.36$ & 62:36: 7.20 & -2.56 & -3.24 & 1.100 & 8.34 & $\ldots$ & 12.5 & 1 & 25 & $\ldots$ \\
\hline Stock 21 & 00:30:14.64 & $57: 55: 51.60$ & -1.07 & -2.76 & 1.700 & 8.82 & $\ldots$ & 4.1 & 3 & 9 & $\ldots$ \\
\hline NGC 381 & 01:08:25.92 & $61: 34: 44.40$ & 0.22 & -2.07 & 1.200 & 8.85 & 0.029 & 9.7 & 0 & 21 & $\ldots$ \\
\hline Stock 3 & 01:12:09.12 & $62: 15: 28.80$ & -0.13 & -0.64 & 1.363 & 7.85 & $\ldots$ & 2.6 & 0 & 3 & $\ldots$ \\
\hline ASCC 6 & 01:47:16.80 & $57: 40: 48.00$ & -0.52 & -0.68 & 1.350 & 7.80 & $\ldots$ & 13.1 & 0 & 26 & $\ldots$ \\
\hline NGC 744 & 01:58:34.80 & $55: 28: 40.80$ & -0.30 & -3.92 & 1.382 & 8.38 & $\ldots$ & 17.4 & 3 & 49 & $\ldots$ \\
\hline NGC 743 & 01:58:37.20 & $60: 09: 25.20$ & -1.81 & -2.77 & 1.100 & 8.29 & 0.090 & 55.8 & 4 & 275 & $\ldots$ \\
\hline ASCC 7 & 01:59:06.00 & $58: 55: 30.00$ & 1.94 & -1.21 & 2.000 & 7.40 & $\ldots$ & 24.9 & 0 & 161 & $\ldots$ \\
\hline Czernik 6 & 02:01:55.20 & $62: 51: 00.00$ & -3.85 & 0.05 & 1.391 & 7.98 & $\ldots$ & 3.9 & 0 & 3 & $\ldots$ \\
\hline Stock 5 & 02:04:32.64 & $64: 21: 18.00$ & 1.12 & -1.16 & 1.100 & 7.70 & $\ldots$ & 5.0 & 0 & 8 & $\ldots$ \\
\hline NGC 869 & 02:18:57.60 & 57:08:42.00 & -2.81 & 0.05 & 2.300 & 7.28 & $\cdots$ & 20.7 & $14 / 15$ & 498 & (d) \\
\hline Basel 10 & $02: 19: 29.52$ & $58: 18: 14.40$ & -0.75 & 1.62 & 2.000 & 7.60 & $\ldots$ & 8.7 & 1 & 16 & $\ldots$ \\
\hline NGC 884 & $02: 22: 02.88$ & $57: 08: 42.00$ & -4.00 & 1.65 & 2.345 & 7.20 & $\ldots$ & 13.9 & $12-13$ & 253 & (d) \\
\hline NGC 886 & $02: 23: 26.88$ & $63: 45: 18.00$ & -1.47 & 0.52 & 1.000 & 8.75 & $\ldots$ & 24.4 & 1 & 43 & $\ldots$ \\
\hline Czernik 8 & 02:33:01.20 & $58: 46: 04.80$ & -0.60 & -0.26 & 1.410 & 8.18 & $\ldots$ & 6.3 & 0 & 6 & $\ldots$ \\
\hline NGC 957 & 02:33:30.48 & $57: 34: 22.80$ & -1.01 & 1.39 & 1.900 & 7.25 & $\ldots$ & 10.0 & $1 / 2,4$ & 48 & (e), (f) \\
\hline Czernik 12 & $02: 39: 22.32$ & $54: 54: 46.80$ & -0.78 & -0.34 & 2.000 & 9.10 & $\ldots$ & 3.0 & 0 & 4 & $\ldots$ \\
\hline SAI 24 & 02:59:27.60 & $60: 32: 60.00$ & -1.42 & -0.20 & 1.000 & 7.20 & $\ldots$ & 9.7 & 0 & 17 & $\ldots$ \\
\hline NGC 1220 & 03:11:41.28 & $53: 20: 49.20$ & 0.30 & 0.40 & 2.100 & 8.20 & $\cdots$ & 3.0 & $0 / 1$ & 3 & (j) \\
\hline King 5 & 03:14:46.32 & $52: 41: 49.20$ & 0.60 & 0.40 & 2.200 & 9.09 & 0.020 & 6.5 & 2 & 10 & $\ldots$ \\
\hline Czernik 15 & 03:23:12.00 & $52: 13: 58.80$ & 1.42 & -0.01 & 2.500 & 7.30 & $\ldots$ & 4.0 & 0 & 9 & $\ldots$ \\
\hline NGC 1348 & 03:34:12.00 & $51: 23: 60.00$ & 1.92 & -3.35 & 1.800 & 8.45 & $\ldots$ & 6.5 & 0 & 11 & $\ldots$ \\
\hline Tombaugh 5 & 03:48:03.60 & 59:04:12.00 & 1.20 & -1.42 & 1.350 & 8.85 & 0.014 & 15.5 & 0 & 47 & $\ldots$ \\
\hline FSR 0663 & 04:06:10.80 & $51: 54: 36.00$ & -3.33 & 2.00 & 1.700 & 8.91 & 0.078 & 6.5 & 0 & 4 & $\ldots$ \\
\hline NGC 1502 & 04:07:49.92 & $62: 19: 51.60$ & 0.01 & -0.54 & 1.023 & 7.08 & $\ldots$ & 17.0 & 0 & 36 & $\ldots$ \\
\hline NGC 1513 & 04:09:42.00 & $49: 30: 36.00$ & -1.08 & -3.49 & 1.400 & 8.50 & $\ldots$ & 15.0 & 3 & 43 & $\ldots$ \\
\hline Berkeley 11 & 04:20:27.60 & 44:54:54.00 & -1.73 & -6.53 & 1.964 & 8.70 & $\ldots$ & 5.4 & 1 & 10 & $\ldots$ \\
\hline FSR 0728 & 04:29:52.80 & $38: 30: 00.00$ & -2.04 & -7.40 & 1.816 & 8.26 & 0.100 & 8.0 & 0 & 8 & $\ldots$ \\
\hline Berkeley 67 & 04:37:48.00 & $50: 46: 30.00$ & 0.09 & -1.49 & 2.000 & 9.24 & 0.029 & 10.4 & 0 & 5 & $\ldots$ \\
\hline FSR 0714 & 04:42:46.80 & 41:55:01.20 & -1.42 & -0.20 & 1.819 & 9.06 & $\ldots$ & 5.6 & 0 & 1 & $\ldots$ \\
\hline Berkeley 68 & 04:44:27.60 & $42: 06: 00.00$ & -5.86 & 0.30 & 1.800 & 9.11 & $\ldots$ & 4.3 & 0 & 0 & $\ldots$ \\
\hline FSR 0717 & 04:46:08.40 & $42: 07: 48.00$ & -3.13 & -1.48 & 1.700 & 9.05 & $\ldots$ & 8.3 & 0 & 4 & $\ldots$ \\
\hline NGC 1664 & 04:51:13.92 & $43: 39: 36.00$ & -4.23 & -4.20 & 1.200 & 8.75 & 0.023 & 13.9 & 1 & 39 & $\ldots$ \\
\hline NGC 1778 & 05:07:58.80 & $37: 01: 40.80$ & -0.76 & -7.59 & 1.500 & 8.45 & $\ldots$ & 9.2 & $1 / 3$ & 28 & (g) \\
\hline $\begin{array}{l}\text { IRAS } 05100 \\
\quad+3723\end{array}$ & 05:13:27.12 & $37: 26: 16.80$ & -4.24 & -7.07 & 2.238 & 7.40 & $\cdots$ & 5.2 & 0 & 9 & $\cdots$ \\
\hline Czernik 20 & $05: 20: 27.60$ & $39: 32: 24.00$ & -3.76 & -1.12 & 2.000 & 9.19 & 0.048 & 3.3 & 0 & 1 & $\ldots$ \\
\hline Berkeley 17 & $05: 20: 37.20$ & $30: 35: 24.00$ & 3.90 & -2.31 & 1.800 & 9.60 & $\ldots$ & 9.6 & 0 & 0 & $\ldots$ \\
\hline SAI 47 & 05:23:56.40 & $42: 19: 22.80$ & -2.81 & -2.58 & 1.523 & 9.20 & $\ldots$ & 6.5 & 0 & 0 & $\ldots$ \\
\hline FSR 0777 & 05:27:36.96 & $34: 44: 52.80$ & 1.40 & -4.27 & 1.173 & 7.00 & $\ldots$ & 4.8 & 0 & 11 & $\ldots$ \\
\hline NGC 1907 & 05:28:04.80 & $35: 19: 30.00$ & 0.85 & -5.70 & 1.600 & 8.60 & $\cdots$ & 7.9 & 0 & 37 & $\cdots$ \\
\hline Stock 8 & 05:28:08.40 & $34: 25: 26.40$ & 1.16 & -5.36 & 1.731 & 7.05 & $\ldots$ & 7.5 & 0 & 30 & $\ldots$ \\
\hline FSR 0736 & 05:29:07.44 & $44: 46: 12.00$ & -1.44 & -4.75 & 1.800 & 9.34 & 0.057 & 6.4 & 0 & 0 & $\ldots$ \\
\hline FSR 0749 & 05:32:06.72 & 41:37:04.80 & 3.19 & 1.13 & 2.300 & 8.62 & 0.071 & 8.7 & 2 & 8 & $\ldots$ \\
\hline NGC 1960 & 05:36:19.68 & $34: 09: 28.80$ & 0.91 & -4.35 & 1.200 & 7.57 & 0.078 & 15.5 & $0 / 5$ & 60 & (h) \\
\hline Koposov 36 & 05:36:47.76 & $31: 11: 60.00$ & 2.94 & -8.86 & 1.600 & 8.45 & 0.021 & 6.3 & 0 & 4 & $\cdots$ \\
\hline FSR 0816 & 05:39:19.68 & $31: 30: 57.60$ & 1.40 & -8.85 & 2.220 & 7.80 & $\ldots$ & 8.8 & 1 & 7 & $\ldots$ \\
\hline Koposov 27 & 05:39:33.12 & $33: 21: 28.80$ & 2.75 & -3.62 & 2.500 & 8.76 & 0.052 & 4.2 & 0 & 2 & $\ldots$ \\
\hline FSR 0799 & 05:42:16.08 & $33: 39: 36.00$ & 0.08 & -3.74 & 2.473 & 9.10 & $\cdots$ & 14.1 & 1 & 34 & $\cdots$ \\
\hline FSR 0826 & 05:42:52.80 & $28: 57: 25.20$ & 2.19 & -2.18 & 2.100 & 7.30 & $\ldots$ & 7.6 & 0 & 7 & $\ldots$ \\
\hline FSR 0850 & 05:45:07.20 & $24: 44: 16.80$ & 0.07 & -3.88 & 1.836 & 7.30 & $\ldots$ & 15.8 & 0 & 42 & $\ldots$ \\
\hline Koposov 10 & $05: 47: 30.00$ & $35: 25: 08.40$ & 4.47 & -4.56 & 1.819 & 8.78 & $\ldots$ & 4.2 & 0 & 3 & $\ldots$ \\
\hline Czernik 23 & 05:50:06.00 & $28: 53: 60.00$ & -2.11 & -2.32 & 2.449 & 8.48 & $\ldots$ & 3.6 & 0 & 6 & $\ldots$ \\
\hline FSR 0852 & $05: 53: 29.28$ & $25: 10: 22.80$ & 3.16 & -0.97 & 2.149 & 9.00 & $\ldots$ & 7.5 & 0 & 6 & $\ldots$ \\
\hline Koposov 12 & 06:01:02.64 & $35: 16: 37.20$ & 0.92 & -1.80 & 1.900 & 8.91 & $\ldots$ & 9.3 & 0 & 23 & $\ldots$ \\
\hline NGC 2129 & 06:01:06.24 & $23: 20: 24.00$ & 1.89 & -1.43 & 1.651 & 7.48 & $\ldots$ & 8.5 & $0 / 2$ & 20 & (b), (c) \\
\hline IC 2157 & 06:04:49.68 & 24:04:12.00 & -0.54 & -3.01 & 2.311 & 7.90 & $\cdots$ & 4.5 & 0 & 16 & $\ldots$ \\
\hline FSR 0833 & $06: 05: 24.00$ & $30: 47: 34.80$ & -0.43 & -4.15 & 2.088 & 9.10 & 0.029 & 6.5 & 0 & 0 & $\cdots$ \\
\hline FSR 0942 & 06:05:58.80 & $13: 39: 36.00$ & -0.98 & -1.77 & 2.082 & 9.00 & $\ldots$ & 4.1 & 0 & 3 & $\ldots$ \\
\hline FSR 0904 & 06:07:03.12 & 19:01:19.20 & 1.63 & -6.72 & 1.427 & 7.80 & $\ldots$ & 7.0 & 1 & 12 & $\ldots$ \\
\hline
\end{tabular}


Table 1

(Continued)

\begin{tabular}{|c|c|c|c|c|c|c|c|c|c|c|c|}
\hline Name & $\begin{array}{c}\text { R.A. } \\
\text { (hh:mm:ss.ss) }\end{array}$ & $\begin{array}{c}\text { Decl. } \\
\text { (dd:mm:ss.ss) }\end{array}$ & $\begin{array}{c}\mu_{\alpha} \cos \delta \\
\left(\operatorname{mas~yr}^{-1}\right)\end{array}$ & $\begin{array}{c}\mu_{\delta} \\
\left(\text { mas yr }^{-1}\right)\end{array}$ & $\begin{array}{l}\text { Distance } \\
(\mathrm{kpc})\end{array}$ & $\begin{array}{c}\log \\
(t \\
\text { (year)) }\end{array}$ & $\begin{array}{l}\text { Uncert. } \\
\text { (years) }\end{array}$ & $\begin{array}{c}\text { Size } \\
(\operatorname{arcmin})\end{array}$ & $\begin{array}{r}\mathrm{N}(\mathrm{Be}) / \\
\mathrm{K}(\mathrm{Be})\end{array}$ & $\begin{array}{l}\mathrm{N}(\mathrm{Be} \\
+\mathrm{B}- \\
\text { type })\end{array}$ & References \\
\hline FSR 0858 & 06:09:10.08 & $25: 40: 48.00$ & 0.52 & -1.48 & 2.127 & 9.01 & 0.057 & 3.9 & 0 & 2 & $\cdots$ \\
\hline NGC 2175 & 06:09:41.28 & 20:30:18.00 & 1.92 & -7.55 & 1.636 & 7.00 & $\cdots$ & 57.1 & $0 / 1$ & 515 & (a) \\
\hline FSR 0952 & $06: 10: 17.28$ & 13:01:01.20 & -1.16 & -1.84 & 1.994 & 9.11 & 0.025 & 18.4 & 0 & 16 & $\ldots$ \\
\hline Pismis 27 & $06: 10: 55.20$ & $20: 36: 54.00$ & 0.98 & -4.03 & 1.664 & 8.25 & 0.203 & 4.0 & 0 & 9 & $\ldots$ \\
\hline FSR 0939 & $06: 10: 58.08$ & $14: 23: 45.60$ & 0.16 & 1.54 & 2.397 & 8.71 & $\cdots$ & 8.5 & 0 & 12 & $\cdots$ \\
\hline FSR 0968 & $06: 11: 21.84$ & 11:52:15.60 & -0.40 & -1.80 & 2.187 & 8.52 & 0.045 & 8.1 & 0 & 27 & $\cdots$ \\
\hline FSR 0956 & $06: 12: 25.20$ & 13:01:12.00 & -1.50 & -0.26 & 2.180 & 8.10 & $\cdots$ & 5.3 & 0 & 4 & $\cdots$ \\
\hline FSR 0940 & $06: 14: 14.88$ & $14: 48: 00.00$ & -0.11 & 1.25 & 2.421 & 9.40 & $\cdots$ & 5.7 & 0 & 8 & $\cdots$ \\
\hline Kronberger 12 & $06: 14: 14.88$ & $22: 28: 58.80$ & -1.04 & -5.85 & 1.893 & 8.87 & 0.084 & 4.0 & 1 & 2 & $\ldots$ \\
\hline BDSB 82 & $06: 14: 59.28$ & $12: 20: 16.80$ & 0.17 & 0.07 & 1.934 & 7.92 & 0.151 & 4.7 & 0 & 4 & $\ldots$ \\
\hline FSR 0891 & 06:17:34.08 & $22: 25: 37.20$ & -0.46 & -5.32 & 2.075 & 8.37 & $\cdots$ & 3.0 & 0 & 5 & $\cdots$ \\
\hline FSR 0902 & 06:18:18.00 & $20: 32: 24.00$ & -0.51 & -5.50 & 1.243 & 7.14 & $\cdots$ & 11.9 & 1 & 21 & $\cdots$ \\
\hline FSR 0953 & $06: 19: 02.16$ & $14: 07: 48.00$ & -0.21 & 2.92 & 1.734 & 8.75 & 0.082 & 4.3 & 0 & 1 & $\ldots$ \\
\hline $\begin{array}{l}\text { Skiff J0619 } \\
\quad+18.5\end{array}$ & $06: 19: 21.84$ & $18: 30: 36.00$ & -0.78 & -3.00 & 1.380 & 7.90 & $\cdots$ & 19.3 & 8 & 50 & $\cdots$ \\
\hline FSR 0951 & $06: 22: 20.16$ & $14: 42: 18.00$ & -0.26 & -0.84 & 1.619 & 8.70 & $\cdots$ & 6.6 & 0 & 21 & $\cdots$ \\
\hline Dolidze 22 & $06: 23: 14.16$ & $04: 35: 24.00$ & -1.14 & -1.42 & 1.775 & 8.37 & $\cdots$ & 5.3 & 1 & 6 & $\cdots$ \\
\hline FSR 0955 & $06: 23: 50.88$ & $14: 30: 54.00$ & 0.90 & 0.02 & 1.994 & 7.40 & $\cdots$ & 4.3 & 2 & 7 & $\cdots$ \\
\hline FSR 1033 & $06: 28: 20.88$ & 02:56:06.00 & 0.59 & -3.52 & 1.710 & 8.52 & $\cdots$ & 6.5 & 0 & 3 & $\cdots$ \\
\hline Juchert 18 & $06: 31: 27.12$ & $06: 48: 14.40$ & -1.81 & -2.04 & 2.290 & 9.02 & 0.040 & 3.4 & 0 & 2 & $\cdots$ \\
\hline NGC 2244 & $06: 31: 55.20$ & 04:56:24.00 & -0.84 & -0.94 & 1.532 & 6.70 & $\cdots$ & 16.2 & 1 & 59 & $\cdots$ \\
\hline FSR 0974 & $06: 32: 34.80$ & $12: 32: 34.80$ & 1.05 & -1.16 & 2.308 & 8.91 & 0.031 & 3.6 & 0 & 4 & $\cdots$ \\
\hline FSR 0905 & $06: 33: 42.48$ & $22: 17: 16.80$ & 0.35 & -4.59 & 1.786 & 8.30 & 0.064 & 5.8 & 3 & 10 & $\cdots$ \\
\hline Basel 8 & 06:34:12.00 & 08:00:36.00 & -2.04 & -0.47 & 1.432 & 7.92 & 0.099 & 27.0 & 3 & 103 & $\cdots$ \\
\hline Collinder 107 & $06: 38: 30.24$ & 04:38:06.00 & -2.50 & -0.26 & 1.447 & 7.17 & 0.016 & 3.6 & 0 & 5 & $\cdots$ \\
\hline Collinder 110 & 06:38:42.00 & 02:02:06.00 & 2.28 & -2.55 & 2.362 & 9.22 & 0.010 & 19.8 & 7 & 47 & $\cdots$ \\
\hline NGC 2269 & $06: 43: 12.00$ & $04: 36: 54.00$ & -0.50 & -2.00 & 1.585 & 8.66 & $\cdots$ & 7.2 & 0 & 15 & $\cdots$ \\
\hline Alessi 16 & $06: 43: 33.60$ & 02:10:30.00 & -0.98 & -2.14 & 2.088 & 8.65 & $\cdots$ & 4.4 & 0 & 11 & $\cdots$ \\
\hline FSR 1025 & $06: 50: 28.80$ & $06: 35: 60.00$ & -1.65 & -1.70 & 2.095 & 8.60 & $\cdots$ & 3.9 & 0 & 3 & $\cdots$ \\
\hline FSR 1029 & 06:54:02.40 & $06: 43: 30.00$ & 0.78 & -3.98 & 1.905 & 8.93 & $\cdots$ & 6.1 & 0 & 5 & $\cdots$ \\
\hline FSR 0866 & 06:55:14.40 & $29: 43: 48.00$ & -1.19 & -1.00 & 1.664 & 9.20 & $\cdots$ & 8.2 & 0 & 2 & $\cdots$ \\
\hline FSR 1147 & 08:00:19.20 & 01:15:36.00 & -3.30 & 2.93 & 1.508 & 8.72 & 0.016 & 14.9 & 0 & 7 & $\cdots$ \\
\hline NGC 6811 & $19: 37: 22.08$ & $46: 23: 42.00$ & -5.68 & -6.70 & 1.227 & 8.80 & 0.017 & 3.6 & 0 & 9 & $\cdots$ \\
\hline NGC 6866 & 20:03:56.88 & 44:09:36.00 & -2.75 & -4.80 & 1.326 & 8.64 & 0.027 & 12.7 & 4 & 31 & $\cdots$ \\
\hline Dolidze 1 & 20:09:42.48 & $36: 29: 52.80$ & -1.59 & 0.09 & 1.445 & 7.60 & $\cdots$ & 6.4 & 0 & 0 & $\cdots$ \\
\hline NGC 6910 & $20: 23: 15.12$ & $40: 47: 42.00$ & -4.27 & -5.72 & 1.127 & 7.53 & 0.026 & 40.1 & $1 / 1$ & 45 & (i) \\
\hline FSR 0213 & 20:26:59.04 & $38: 58: 19.20$ & -2.59 & -3.73 & 1.434 & 8.71 & 0.065 & 8.4 & 0 & 9 & $\cdots$ \\
\hline Patchick 103 & 20:34:55.92 & $53: 17: 45.60$ & -4.94 & -5.20 & 1.332 & 8.95 & $\cdots$ & 6.3 & 0 & 0 & $\cdots$ \\
\hline CBJC 4 & $20: 56: 17.52$ & $44: 23: 24.00$ & -6.78 & -3.67 & 1.293 & 7.70 & $\cdots$ & 12.3 & 0 & 12 & $\cdots$ \\
\hline Collinder 428 & 21:03:04.32 & $44: 33: 36.00$ & -5.81 & -1.78 & 1.185 & 8.86 & $\cdots$ & 6.4 & 0 & 3 & $\cdots$ \\
\hline FSR 0253 & 21:03:30.72 & $39: 58: 48.00$ & -3.72 & -0.62 & 1.414 & 8.28 & $\cdots$ & 13.6 & 7 & 14 & $\cdots$ \\
\hline NGC 7031 & 21:07:06.00 & $50: 52: 30.00$ & -1.95 & -4.01 & 1.100 & 8.20 & $\ldots$ & 49.1 & 0 & 95 & $\ldots$ \\
\hline FSR 0354 & $22: 11: 09.60$ & $57: 39: 36.00$ & -2.80 & -3.64 & 1.169 & 8.00 & $\cdots$ & 14.4 & 2 & 33 & $\cdots$ \\
\hline FSR 0398 & $22: 36: 14.40$ & $64: 07: 58.80$ & -2.37 & -0.45 & 1.200 & 7.70 & $\cdots$ & 20.9 & 0 & 23 & $\ldots$ \\
\hline NGC 7654 & $23: 24: 42.48$ & $61: 38: 13.20$ & -0.23 & -3.50 & 1.350 & 7.90 & $\cdots$ & 20.7 & 2 & 132 & $\cdots$ \\
\hline
\end{tabular}

Note. Column 1: Names of clusters. Column 2: Right Ascension (R.A.) in hms (J2000). Column 3: Declination (decl.) in hms (J2000). Column 4: Proper motion of R.A. Column 5: Proper motion of decl. Column 6: Distance of clusters to the Sun. Column 7: Logarithm of the cluster age (t). Column 8: Uncertainties of ages in logarithm. Column 9: Size of searching region. Column 10: Numbers of Be star candidates of clusters found in this work (N(Be)). The notation $\mathrm{K}(\mathrm{Be})$ is known Be star numbers from literature. Column 11: Numbers of B-type stars in clusters. Column 12: References of known Be stars: (a) Zhang et al. (2008), (b) Morgan et al. (1955), (c) WEBDA: https://www.univie.ac.at/webda/; (d) Marsh Boyer et al. (2012), (e) Schild \& Romanishin (1976), (f) Mathew et al. (2008), (g) Barbon \& Hassan (1973), (h) Souza et al. (2016), (i) Kubát et al. (2007), (j) Mathew \& Subramaniam (2011). 

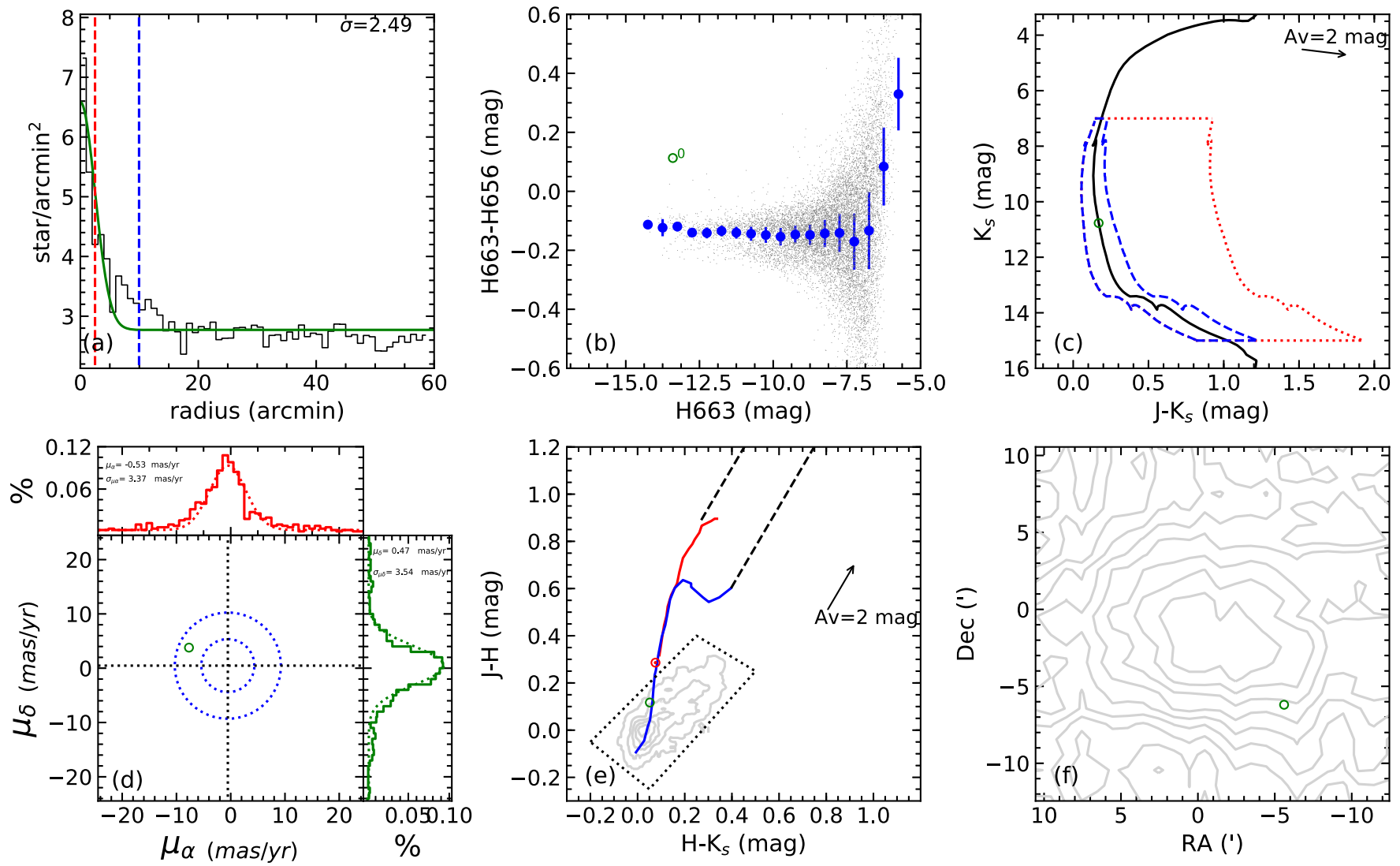

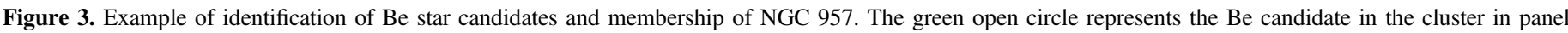

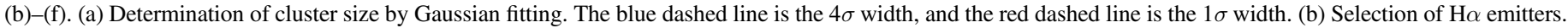

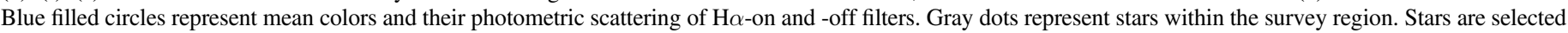

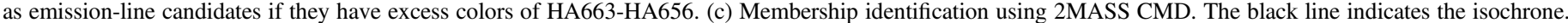

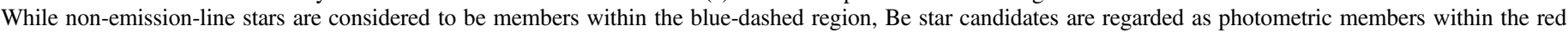

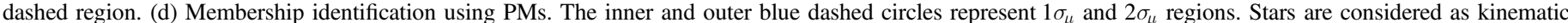

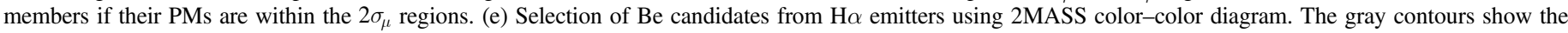

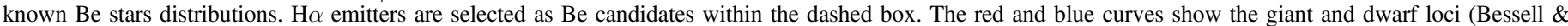

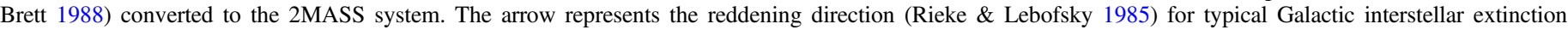
$(R v=3.1)$, and the dashed lines encompass the region of reddened giants and dwarfs. (f) Spatial distribution of cluster and Be candidates.

\section{Data Analysis and Method}

\subsection{Determination of the Searching Region}

We used the radial density profile to determine the searching region (Figure 3(a)). We have selected stars from the PPMXL $^{12}$ (Roeser et al. 2010) data with a signal-to-noise ratio $(\mathrm{S} / \mathrm{N}) \geqslant 10$ in the Two Micron All Sky Survey (2MASS; Cutri et al. 2003) $J, H$, and $K_{s}$ bands. Because $10 \%$ of objects of PPNXL include spurious entries (Roeser et al. 2010), Kharchenko et al. (2012) have averaged and computed their PMs and errors. Here, we used these PMs instead of PPMXL data. Then we used the half-Gaussian fitting of the radial density profile to determine a $3 \sigma$ radius. As some open clusters have irregular shapes due to their evolution processes, we adopted a box with the side of $8 \sigma(2 \times 4 \sigma)$ region as the searching region for these open clusters.

\subsection{Searching for Emission-line Candidates}

We used differential photometry with $\mathrm{H} \alpha$-on and -off images from $\mathrm{PTF} / \mathrm{iPTF}$ to identify $\mathrm{H} \alpha$ emission-line candidates (Figure 3(b)). The $\mathrm{H} \alpha$-on and -off images were taken through

\footnotetext{
12 http://vizier.u-strasbg.fr/viz-bin/VizieR?-source $=\% 20 \mathrm{PPMXL}$
}

HA656 and HA663 narrow-band filters (Law et al. 2009) and were processed for bias corrections, flat fielding, and astrometric calibration (Laher et al. 2014). As the $\mathrm{H} \alpha$ emitters can exhibit excess colors of HA663-HA656 (e.g., flux excess in HA656 image), we selected candidates that stand out in the HA663-HA656 colors. We adopted different selection criteria for stars with different brightness because faint stars have increased scattering of the HA663-HA656 colors due to photometric errors. We first calculated the photometric scattering $\left(\sigma_{p}\right)$ of HA663-HA656 colors within each $0.5 \mathrm{mag}$ bin of HA663. Then we selected probable $\mathrm{H} \alpha$ emitters for those with HA663-HA656 $>2 \sigma_{p}+$ mean $_{\text {color }}$, whereas mean $_{\text {color }}$ is the mean of HA663-HA656 colors within each $0.5 \mathrm{mag}$ bin of HA663. We only show the emission-line candidates after membership identification in Figure 3(b) (green open circle).

\subsection{Identifying Photometric Membership}

To characterize the membership of emission-line star candidates, we used near-infrared (NIR) photometric data of 2MASS that provides a $10 \sigma$ detection limit in the $J, H$, and $K_{s}$ bands. We defined a region of photometric membership by estimating $J-K_{s}$ errors propagated from photometric errors of 
Table 2

Basic Properties of Be Star Candidates

\begin{tabular}{|c|c|c|c|c|c|c|c|c|}
\hline Object & $\begin{array}{c}\text { R.A. } \\
\text { (hh:mm:ss.ss) }\end{array}$ & $\begin{array}{c}\text { Decl. } \\
\text { (dd:mm:ss.ss) }\end{array}$ & $\begin{array}{c}\mu_{\alpha} \cos \delta \\
\left(\mathrm{mas} \mathrm{yr}^{-1}\right)\end{array}$ & $\begin{array}{c}\mu_{\delta} \\
\left(\operatorname{mas~yr}^{-1}\right)\end{array}$ & $\begin{array}{c}J-H \\
(\mathrm{mag})\end{array}$ & $\begin{array}{l}H-K_{s} \\
(\mathrm{mag})\end{array}$ & $\begin{array}{c}R \\
(\mathrm{mag})\end{array}$ & SIMBAD \\
\hline Stock 20-1 & $00: 25: 27.12$ & $62: 42: 37.13$ & -3.0 & 0.1 & 0.037 & 0.109 & 11.400 & $\cdots$ \\
\hline Stock 21-3 & 00:30:06.16 & 57:56:01.97 & -2.6 & -1.5 & 0.171 & 0.067 & 13.160 & $\cdots$ \\
\hline Stock 21-2 & 00:30:09.54 & $57: 56: 33.12$ & -6.9 & -1.8 & 0.116 & 0.113 & 13.370 & $\cdots$ \\
\hline Stock 21-1 & 00:30:23.97 & $57: 56: 30.29$ & -1.3 & 0.8 & 0.109 & 0.104 & 12.770 & $\ldots$ \\
\hline NGC 743-4 & 01:58:01.31 & $60: 56: 45.61$ & -6.3 & 3.3 & 0.263 & 0.174 & 13.070 & $\cdots$ \\
\hline NGC 744-13 & 01:58:11.73 & $55: 42: 18.68$ & 0.2 & 6.8 & 0.208 & 0.092 & 11.230 & $\ldots$ \\
\hline NGC 743-3 & 01:58:21.22 & 59:53:59.89 & -8.1 & 2.2 & 0.201 & 0.146 & 13.370 & $\cdots$ \\
\hline NGC 744-1 & 01:59:29.50 & $55: 26: 23.48$ & -0.5 & -4.7 & 0.035 & 0.043 & 11.470 & $\ldots$ \\
\hline NGC 744-5 & 01:59:33.00 & $55: 29: 34.02$ & 2.6 & -8.8 & 0.152 & 0.065 & 12.680 & $\ldots$ \\
\hline NGC 743-1 & 02:00:10.06 & $60: 10: 53.49$ & 1.6 & -1.0 & 0.285 & 0.298 & 11.350 & $\mathrm{Be}$ \\
\hline NGC 743-2 & 02:00:31.68 & 59:53:58.97 & 5.8 & 3.2 & 0.120 & 0.049 & 11.950 & $\ldots$ \\
\hline NGC 869-2 & $02: 16: 47.63$ & $57: 17: 38.52$ & 1.6 & 3.0 & 0.158 & 0.089 & 12.210 & $\ldots$ \\
\hline NGC 869-1 & $02: 17: 30.82$ & $57: 17: 47.30$ & 2.0 & -3.2 & 0.106 & 0.051 & 12.110 & $\mathrm{iC}$ \\
\hline NGC 869-4 & $02: 18: 18.73$ & $57: 22: 01.75$ & -3.9 & -5.4 & 0.227 & 0.164 & 14.240 & $\mathrm{iC}$ \\
\hline NGC 869-3 & $02: 18: 24.29$ & $57: 21: 42.26$ & -6.3 & -2.9 & 0.175 & 0.109 & 13.890 & $\mathrm{iC}$ \\
\hline NGC 869-16 & 02:19:05.87 & 57:13:57.39 & -2.2 & -6.9 & 0.152 & 0.114 & 12.220 & $\mathrm{iC}$ \\
\hline NGC 869-26* & 02:19:08.63 & 57:03:48.99 & -1.4 & -0.9 & 0.289 & 0.215 & 12.420 & Be (B5) \\
\hline NGC 869-14 & $02: 19: 29.23$ & 57:09:48.19 & 3.5 & 0.1 & 0.102 & 0.046 & 11.650 & $\mathrm{iC}$ \\
\hline NGC 869-17 & $02: 19: 33.22$ & 57:15:08.42 & -5.3 & -3.8 & 0.095 & 0.077 & 12.370 & $\mathrm{iC}$ \\
\hline NGC 869-18 & 02:19:58.29 & $57: 10: 15.42$ & -6.7 & 3.3 & 0.185 & 0.140 & 13.670 & $\mathrm{iC}$ \\
\hline NGC 869-20 & $02: 19: 59.28$ & $57: 21: 43.78$ & -1.2 & -2.1 & 0.152 & 0.139 & 14.640 & $\ldots$ \\
\hline NGC 886-1 & 02:20:03.78 & $63: 37: 24.80$ & -3.2 & 3.0 & 0.344 & 0.273 & 13.400 & Em \\
\hline NGC 869-15 & 02:20:04.05 & $57: 03: 19.72$ & -5.0 & 2.9 & 0.106 & 0.050 & 11.760 & $\mathrm{iC}$ \\
\hline NGC 869-25 & 02:20:07.68 & $57: 18: 56.18$ & -0.6 & 10.3 & 0.177 & 0.129 & 13.210 & iC \\
\hline Basel 10-1 & $02: 20: 20.34$ & $58: 19: 32.51$ & 0.4 & 6.5 & 0.220 & 0.331 & 11.690 & $\mathrm{Be}$ \\
\hline NGC 884-2 & 02:20:57.89 & $57: 18: 27.55$ & -1.7 & -2.6 & 0.122 & 0.055 & 11.390 & iC \\
\hline NGC 869-19 & $02: 21: 03.28$ & $56: 59: 49.67$ & -7.0 & -0.8 & 0.272 & 0.138 & 14.260 & $\mathrm{iC}$ \\
\hline NGC 884-14 & $02: 21: 03.28$ & $56: 59: 49.67$ & -7.0 & -0.8 & 0.272 & 0.138 & 14.260 & iC \\
\hline NGC 869-27* & $02: 21: 24.91$ & $57: 11: 52.57$ & -5.7 & 8.3 & 0.196 & 0.215 & 12.186 & $\mathrm{Be}(\mathrm{B} 2)$ \\
\hline NGC 884-19* & $02: 21: 24.91$ & $57: 11: 52.57$ & -5.7 & 8.3 & 0.196 & 0.215 & 12.190 & $\mathrm{Be}(\mathrm{B} 2)$ \\
\hline NGC 884-11 & $02: 21: 33.88$ & $56: 56: 15.72$ & -4.4 & -4.8 & 0.170 & 0.094 & 12.960 & $\mathrm{iC}$ \\
\hline NGC 884-6 & $02: 21: 46.02$ & $57: 11: 26.42$ & -4.9 & 2.0 & 0.067 & 0.129 & 13.520 & $\mathrm{iC}$ \\
\hline NGC 884-1 & $02: 21: 46.43$ & $57: 14: 07.81$ & 3.1 & -1.3 & 0.174 & 0.074 & 11.290 & $\mathrm{iC}$ \\
\hline NGC 884-12 & $02: 21: 47.92$ & $57: 15: 21.27$ & -3.7 & 1.2 & 0.199 & 0.132 & 13.310 & $\mathrm{iC}$ \\
\hline NGC 884-8 & 02:22:10.08 & $57: 03: 02.85$ & -5.8 & -8.1 & 0.090 & 0.036 & 12.260 & $\mathrm{iC}$ \\
\hline NGC 884-13 & $02: 22: 10.18$ & $57: 20: 53.83$ & -4.8 & -1.1 & 0.211 & 0.087 & 14.360 & $\mathrm{iC}$ \\
\hline NGC 884-4 & $02: 22: 23.04$ & $57: 03: 48.56$ & -6.6 & 2.8 & 0.083 & 0.040 & 12.370 & $\mathrm{iC}$ \\
\hline NGC 884-3 & 02:23:07.12 & $57: 04: 54.54$ & -5.7 & 4.2 & 0.134 & 0.085 & 12.700 & $\mathrm{iC}$ \\
\hline NGC 884-5 & 02:23:19.90 & $57: 17: 20.91$ & -3.3 & 3.0 & 0.227 & 0.127 & 13.250 & $\mathrm{iC}$ \\
\hline NGC 957-1* & 02:33:08.19 & $57: 28: 10.88$ & -7.7 & 3.8 & 0.117 & 0.052 & 11.650 & $\mathrm{Be}$ \\
\hline King 5-1 & 03:14:21.74 & $52: 40: 47.19$ & -0.4 & 1.3 & 0.194 & 0.151 & 13.540 & $\cdots$ \\
\hline King 5-3 & 03:15:19.71 & $52: 38: 00.72$ & -1.3 & 0.4 & 0.223 & 0.164 & 15.150 & $\cdots$ \\
\hline NGC 1513-3 & 04:09:43.44 & $49: 28: 42.55$ & -2.6 & -2.7 & 0.290 & 0.177 & 13.450 & $\mathrm{iC}$ \\
\hline NGC 1513-1 & 04:10:08.22 & $49: 26: 54.55$ & -0.5 & -6.1 & 0.142 & 0.132 & 12.330 & $\mathrm{iC}$ \\
\hline NGC 1513-2 & 04:10:17.53 & $49: 27: 49.96$ & -1.3 & -8.9 & 0.166 & 0.071 & 12.820 & $\mathrm{iC}$ \\
\hline Berkeley 11-1 & $04: 20: 26.86$ & $44: 52: 44.79$ & -6.5 & -2.5 & 0.300 & 0.164 & 14.660 & $\ldots$ \\
\hline NGC 1664-1 & 04:52:08.05 & 43:42:07.39 & -1.1 & 0.9 & 0.078 & 0.055 & 12.420 & $\mathrm{iC}$ \\
\hline NGC $1778-1^{*}$ & 05:08:11.74 & $36: 57: 02.85$ & 3.5 & -6.8 & 0.095 & 0.100 & 11.030 & $\cdots$ \\
\hline FSR 0749-2 & 05:31:41.67 & $41: 33: 35.36$ & -0.7 & 0.5 & 0.164 & 0.089 & 13.490 & $\cdots$ \\
\hline FSR 0749-1 & $05: 32: 32.83$ & $41: 35: 53.28$ & 2.5 & -0.9 & 0.090 & 0.071 & 13.400 & $\cdots$ \\
\hline FSR 0816-1 & 05:38:48.06 & $31: 31: 58.17$ & 1.5 & -6.7 & 0.268 & 0.128 & 13.740 & $\cdots$ \\
\hline FSR 0799-1 & $05: 42: 32.10$ & $33: 28: 59.98$ & -1.1 & 3.5 & 0.135 & 0.167 & 14.110 & $\ldots$ \\
\hline FSR 0904-1 & $06: 06: 59.26$ & 19:02:46.91 & -4.6 & -7.6 & 0.111 & 0.131 & 12.450 & $\cdots$ \\
\hline Kronberger 12-1 & $06: 14: 17.82$ & $22: 25: 30.87$ & -4.0 & -4.1 & 0.269 & 0.153 & 12.840 & $\cdots$ \\
\hline Skiff J0619+18.5-13 & $06: 18: 28.07$ & $18: 29: 22.94$ & -3.3 & -1.8 & 0.293 & 0.150 & 13.720 & $\ldots$ \\
\hline Skiff J0619+18.5-12 & $06: 18: 29.97$ & $18: 36: 18.94$ & 4.8 & -4.2 & 0.123 & 0.072 & 12.710 & $\cdots$ \\
\hline Skiff J0619+18.5-11 & $06: 18: 31.20$ & $18: 42: 25.87$ & -7.2 & -4.3 & 0.112 & 0.050 & 12.180 & $\ldots$ \\
\hline FSR 0902-1 & 06:19:04.97 & $20: 35: 21.87$ & -2.2 & -5.3 & 0.172 & 0.033 & 12.690 & $\cdots$ \\
\hline Skiff J0619+18.5-2 & $06: 19: 12.04$ & $18: 29: 47.52$ & -1.4 & -2.3 & 0.042 & 0.107 & 11.840 & $\cdots$ \\
\hline Skiff J0619+18.5-3 & $06: 19: 20.08$ & $18: 13: 23.20$ & -3.4 & -3.5 & 0.016 & 0.088 & 11.740 & $\cdots$ \\
\hline Skiff J0619+18.5-4 & $06: 19: 36.38$ & $18: 25: 41.62$ & -3.4 & -0.2 & 0.187 & 0.134 & 12.870 & $\cdots$ \\
\hline Skiff J0619+18.5-5 & $06: 19: 51.87$ & $18: 12: 52.66$ & -1.9 & -6.2 & 0.237 & 0.110 & 13.080 & $\cdots$ \\
\hline Skiff J0619+18.5-1 & 06:20:01.00 & $18: 36: 26.38$ & -1.0 & -0.1 & 0.079 & 0.111 & 12.170 & $\cdots$ \\
\hline
\end{tabular}


Table 2

(Continued)

\begin{tabular}{|c|c|c|c|c|c|c|c|c|}
\hline Object & $\begin{array}{c}\text { R.A. } \\
\text { (hh:mm:ss.ss) }\end{array}$ & $\begin{array}{c}\text { Decl. } \\
\text { (dd:mm:ss.ss) }\end{array}$ & $\begin{array}{c}\mu_{\alpha} \cos \delta \\
\left(\text { mas yr }^{-1}\right)\end{array}$ & $\begin{array}{c}\mu_{\delta} \\
\left(\operatorname{mas~yr}^{-1}\right)\end{array}$ & $\begin{array}{l}J-H \\
(\mathrm{mag})\end{array}$ & $\begin{array}{l}H-K_{s} \\
(\mathrm{mag})\end{array}$ & $\begin{array}{c}R \\
(\mathrm{mag})\end{array}$ & SIMBAD \\
\hline Dolidze 22-1 & $06: 23: 05.02$ & $04: 34: 12.34$ & -3.9 & -2.2 & 0.166 & 0.131 & 14.130 & $\ldots$ \\
\hline FSR 0955-1 & $06: 23: 50.30$ & $14: 30: 55.57$ & 2.0 & -1.8 & 0.067 & 0.067 & 11.010 & $\ldots$ \\
\hline FSR 0955-2 & $06: 24: 01.29$ & $14: 29: 43.15$ & -2.3 & 2.0 & 0.051 & 0.030 & 12.100 & $\ldots$ \\
\hline NGC 2244-1 & $06: 32: 43.48$ & $04: 51: 21.50$ & -3.3 & 1.8 & 0.116 & 0.109 & 12.240 & $\mathrm{iC}$ \\
\hline FSR 0905-4 & $06: 33: 41.74$ & $22: 16: 19.56$ & 2.3 & -9.1 & 0.092 & 0.053 & 13.260 & $\ldots$ \\
\hline FSR 0905-1 & $06: 33: 49.13$ & $22: 16: 37.40$ & 3.1 & -7.1 & 0.116 & 0.030 & 12.150 & $\ldots$ \\
\hline FSR 0905-2 & $06: 33: 51.78$ & 22:18:41.66 & -0.7 & -7.1 & 0.166 & 0.037 & 13.010 & $\ldots$ \\
\hline Basel 8-1 & $06: 34: 41.48$ & $07: 56: 42.26$ & 0.7 & -2.0 & 0.110 & 0.056 & 11.660 & $\cdots$ \\
\hline Basel 8-3 & 06:34:57.09 & $08: 10: 55.74$ & -0.6 & -3.9 & 0.067 & 0.136 & 12.770 & $\cdots$ \\
\hline Basel 8-2 & $06: 35: 38.31$ & 08:04:13.36 & -8.2 & 0.1 & 0.045 & 0.075 & 11.450 & $\mathrm{iC}$ \\
\hline Collinder 110-3 & $06: 37: 58.50$ & $02: 16: 29.16$ & 4.9 & 0.9 & 0.263 & 0.184 & 14.770 & $\ldots$ \\
\hline Collinder 110-2 & $06: 38: 24.54$ & 02:17:05.31 & 3.3 & -0.3 & 0.174 & 0.068 & 14.040 & $\ldots$ \\
\hline Collinder 110-1 & $06: 38: 38.27$ & $02: 13: 36.76$ & 4.6 & -1.6 & 0.266 & 0.137 & 13.970 & $\mathrm{iC}$ \\
\hline Collinder 110-5 & 06:38:49.79 & 02:08:48.72 & 0.2 & -1.0 & 0.172 & 0.139 & 14.320 & $\mathrm{iC}$ \\
\hline Collinder 110-4 & $06: 38: 54.16$ & 02:11:57.40 & 0.9 & -0.6 & 0.204 & 0.150 & 14.010 & $\ldots$ \\
\hline Collinder 110-12 & $06: 38: 55.36$ & 01:57:02.14 & -5.2 & -6.0 & 0.214 & 0.133 & 14.190 & $\mathrm{iC}$ \\
\hline Collinder 110-6 & $06: 39: 22.49$ & 01:49:33.72 & 7.0 & -0.1 & 0.320 & 0.180 & 14.420 & $\ldots$ \\
\hline NGC 6866-12 & 20:03:04.09 & 44:14:15.69 & -4.6 & -3.3 & 0.082 & 0.041 & 12.600 & $\ldots$ \\
\hline NGC 6866-1 & 20:03:22.23 & $44: 16: 57.64$ & -3.4 & 1.9 & 0.047 & 0.083 & 12.110 & $\mathrm{iC}$ \\
\hline NGC 6866-2 & 20:03:49.51 & 44:10:50.70 & -1.6 & -3.9 & 0.050 & 0.049 & 12.150 & $\mathrm{iC}$ \\
\hline NGC 6866-3 & $20: 03: 56.83$ & $44: 10: 31.90$ & -4.0 & -5.8 & 0.073 & 0.029 & 12.120 & $\ldots$ \\
\hline NGC 6910-1 & 20:22:07.18 & $40: 48: 39.95$ & -3.4 & 0.4 & 0.208 & 0.120 & 12.360 & $\mathrm{iC}$ \\
\hline FSR 0253-2 & $21: 02: 33.46$ & $39: 53: 13.80$ & 3.2 & -2.0 & 0.140 & 0.122 & 11.880 & $\mathrm{iC}$ \\
\hline FSR 0253-6 & 21:03:15.26 & $39: 56: 36.24$ & -10.1 & -5.2 & 0.214 & 0.144 & 12.850 & $\ldots$ \\
\hline FSR 0253-1 & 21:03:23.05 & 39:59:17.95 & 4.5 & -0.4 & 0.075 & 0.107 & 11.100 & $\ldots$ \\
\hline FSR 0253-5 & 21:03:27.60 & 40:05:44.89 & -9.9 & 0.4 & 0.213 & 0.196 & 13.180 & $\ldots$ \\
\hline FSR 0253-4 & 21:03:28.38 & $40: 08: 46.48$ & -4.5 & 2.7 & 0.177 & 0.136 & 13.320 & $\ldots$ \\
\hline FSR 0253-7 & 21:03:57.98 & 40:10:09.00 & -6.0 & -3.6 & 0.335 & 0.215 & 14.050 & $\ldots$ \\
\hline FSR 0253-3 & 21:03:59.37 & 40:01:08.51 & -4.6 & -2.1 & 0.207 & 0.098 & 12.610 & $\ldots$ \\
\hline FSR 0354-1 & $22: 10: 51.21$ & $57: 49: 33.71$ & -9.0 & -3.5 & 0.298 & 0.311 & 11.610 & Em \\
\hline FSR 0354-2 & $22: 12: 13.56$ & $57: 39: 37.34$ & -1.8 & 3.0 & 0.275 & 0.302 & 11.480 & $\ldots$ \\
\hline NGC 7654-1* & $23: 24: 21.86$ & $61: 29: 21.08$ & -3.1 & -6.5 & 0.132 & 0.240 & 11.590 & Em \\
\hline NGC 7654-2* & $23: 24: 46.38$ & $61: 26: 15.41$ & -8.7 & 3.0 & 0.179 & 0.219 & 11.090 & $\mathrm{Be}(\mathrm{B} 9)$ \\
\hline
\end{tabular}

Note. Column 1: Names of Be star candidates. Names with an asterisk are objects reported in WEBDA. Column 2: Right Ascension (R.A.) in hms (J2000). Column 3: Declination (decl.) in hms (J2000). Column 4: Proper motion of R.A. Column 5: Proper motion of decl. Column 6: 2MASS $J-H$ colors. Column 7: 2 MASS $H-K_{s}$ colors. Column 8: $R$-band magnitudes obtained from USNO-B and PTF/iPTF catalogue. Column 9: Object classification adopted from SIMBAD; Be: Be stars; iC: star in cluster; Em: emission-line stars.

$J$ and $K_{s}$ values along with the isochrone from bright toward faint $K_{s}$ magnitudes in the color-magnitude diagram (CMD; blue-dashed lines in Figure 3(c)). The photometric membership is determined by selecting stars within the region that are near the isochrone (Girardi et al. 2002) for non-emission-line stars. However, Be stars could have unusually large infrared excess with $J-H$ and $H-K_{s}$ both being greater than 0.6 mag (Lee $\&$ Chen 2011). Therefore, we extended the selection region for the emission-line candidates with $J-K_{s} \sim 1.2$ mag (reddashed lines in Figure 3(c)).

\subsection{Identifying Kinematic Membership}

After identification of photometric membership, kinematic information can be obtained to further secure the membership in a star cluster by PMs. We have calculated the averaged PM and standard deviations of stars within the searching region by fitting a Gaussian distribution to PMs $\mu \alpha$ and $\mu \beta$. The $\sigma_{\mu}$ is the error propagated from $\sigma_{\mu \alpha}$ and $\sigma_{\mu \beta}$, whereas $\sigma_{\mu \alpha}$ and $\sigma_{\mu \beta}$ are the standard deviations of $\mu \alpha$ and $\mu \beta$, respectively. To exclude stars with inconsistent or high PMs, stars are considered to be kinematic members if they are located inside the $2 \sigma_{\mu}$ region.
It should be noted that our $2 \sigma$ approach is just an approximate method. Better models would be required to obtain more precise determinations of kinematic membership.

\subsection{Identifying Be Star Candidates}

We defined a region in the $J-H$ versus $H-K_{s}$ color-color diagram to identify possible Be star candidates from emissionline candidates under the assumption that Be stars have similar infrared colors. As shown in Figure 3(e), the gray-dashed region covers gray contours representing infrared colors of over 1000 Be stars from the literature (Zhang et al. 2005). We thus selected possible Be star candidates inside the gray-dashed region in the color-color diagram. In Figure 3(f), we show the spatial distribution of clusters and Be star candidates. In Table 2, we list the properties of the Be candidates.

\subsection{Selecting B-type Stars}

We adopted the absolute $K_{s}$-band magnitude range ${ }^{13}$ of B-type stars (-3.04-1.07 mag; see also Pecaut et al. 2012;

\footnotetext{
${ }^{13}$ http://www.pas.rochester.edu/ emamajek/
} 


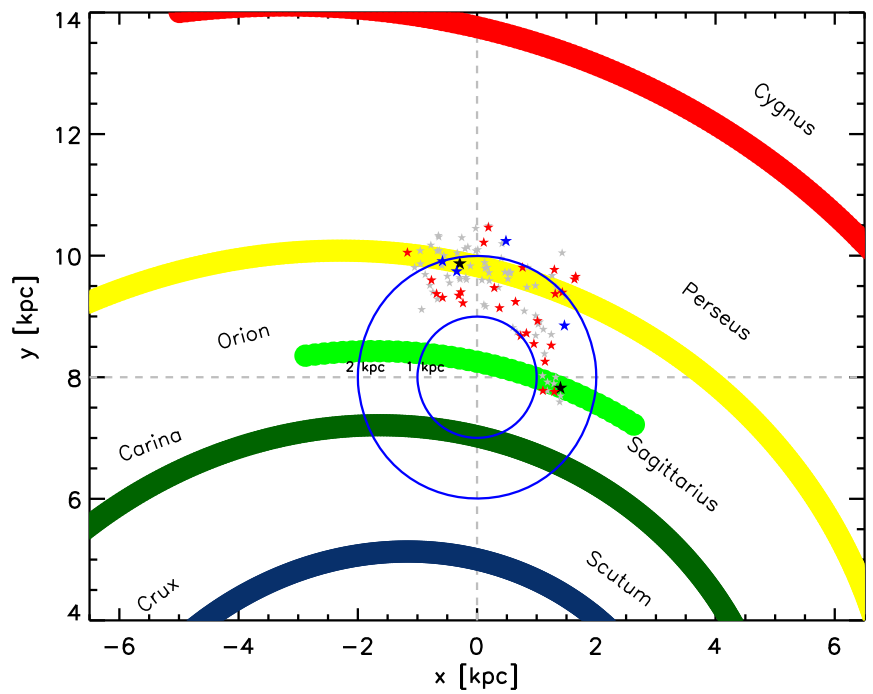

Figure 4. Cluster distribution in Galactic plane. Inner and outer blue circles represent $1 \mathrm{kpc}$ and $2 \mathrm{kpc}$ radii, respectively. Gray stars represent clusters with Be fraction $=0 \%$; red stars show clusters with Be fraction $<20 \%$; blue stars depict clusters with Be fraction $>20 \%$ and $<40 \%$; black stars show clusters with Be fraction $>40 \%$.

Pecaut \& Mamajek 2013). For each cluster, we converted absolute magnitude to apparent magnitudes on the basis of their distance given by Kharchenko et al. (2013). Then we selected the stars that have apparent $K_{s}$-band magnitude within the range of B-type stars.

\section{Results and Discussion}

\subsection{Clusters with Be Star Candidates}

We discovered $85 \mathrm{Be}$ star candidates in 27 clusters among 104 open clusters. The spatial distributions of the 104 clusters in the MW are shown in Figures 4 and 5. Most of the clusters are concentrated in the Galactic plane with latitude $|\mathrm{b}|<5^{\circ}$. The clusters with Be candidates are not only located in spiral arms but also in the regions between arms. Most of clusters with the $\mathrm{Be}$ fraction $\mathrm{N}(\mathrm{Be}) / \mathrm{N}(\mathrm{Be}+\mathrm{B}$-type $)<20 \%$ distribute randomly. However, clusters with Be star fraction $\mathrm{N}(\mathrm{Be}) / \mathrm{N}(\mathrm{Be}$ +B-type) $>20 \%$ tend to be located in spiral arms (blue and black stars in Figure 4). Similarly, Mathew et al. (2008) showed that clusters possessing emission stars lie in the Perseus arm, indicating more active star formation. And yet it should be noted that these behaviors could change, as more data should be included to perform a statistical study. In Figure 6, among 32 clusters with $\mathrm{Be}$ star candidates, more than $50 \%$ of clusters have a Be fraction $\mathrm{N}(\mathrm{Be}) / \mathrm{N}(\mathrm{Be}+\mathrm{B}$-type) less than $10 \%$, which is consistent with previous studies (McSwain \& Gies 2005; Mathew et al. 2008).

In Figure 7, we showed the fractions of clusters with Be star candidates as a function of age, which ranges from millions to billions of years. Each age bin shows a ratio of clusters with Be star candidates over all clusters within corresponding age. This ratio increases with age until $8.0<\log (t($ year $)) \leqslant 8.5$, and then declines all the way to the oldest age bin (solid histogram in Figure 7). However, because our $\mathrm{H} \alpha$ data are limited to $R$-band magnitudes between $\sim 11$ and 16 mag, clusters with different distances would have different Be star completeness. We found that clusters with distance $>1.5 \mathrm{kpc}$ give the completeness of spectral type later than B5. Thus, if we only consider the clusters with distance $>1.5 \mathrm{kpc}$, the peak of the ratios is in the bin of

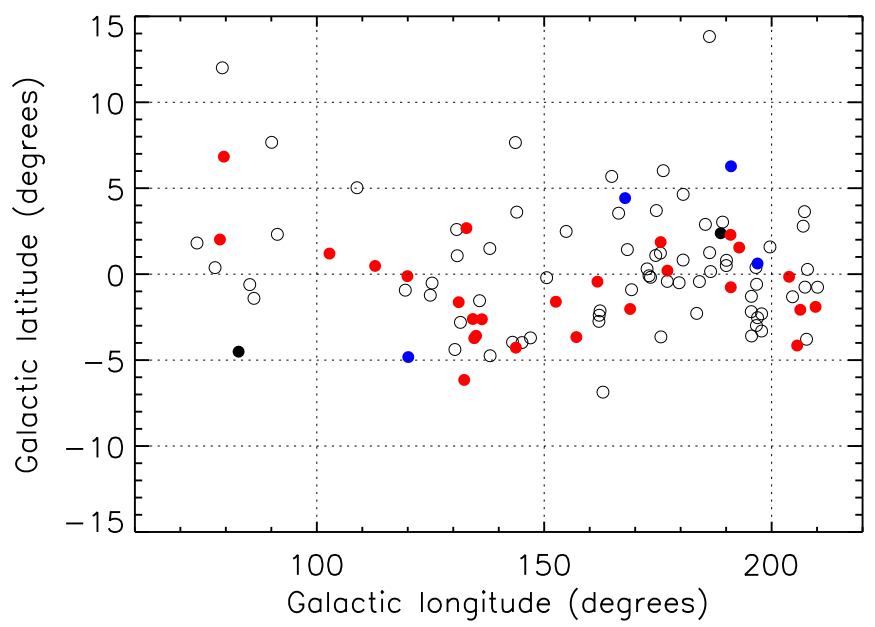

Figure 5. Cluster distribution in Galactic coordinates. Open circles: clusters with Be fraction $=0 \%$; red filled circles: clusters with Be fraction $<20 \%$; blue filled circles: clusters with Be fraction $>20 \%$ and $<40 \%$; black filled circles: clusters with Be fraction $>40 \%$.

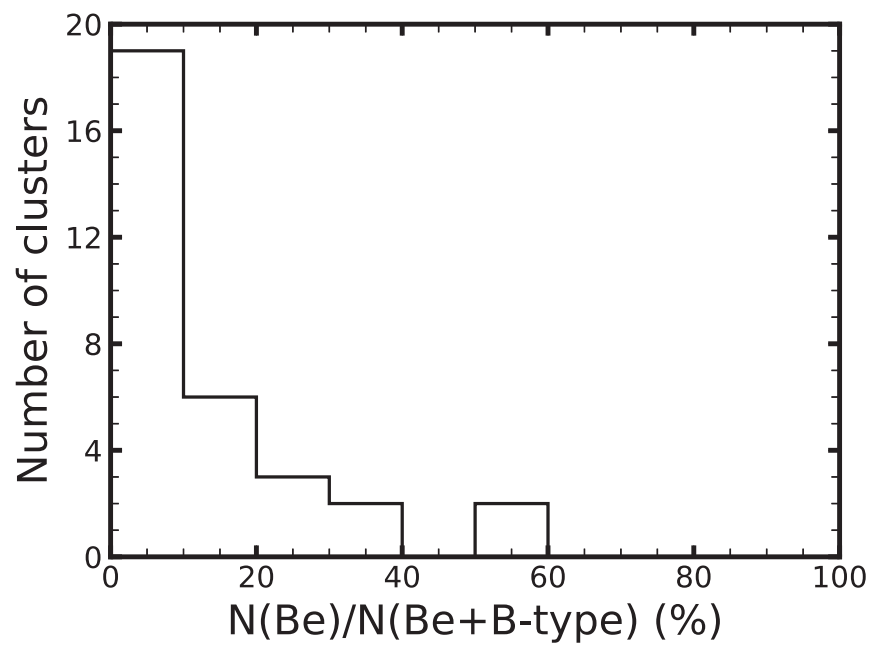

Figure 6. Fraction of clusters with Be star candidates. Most clusters have Be fraction $<20 \%$.

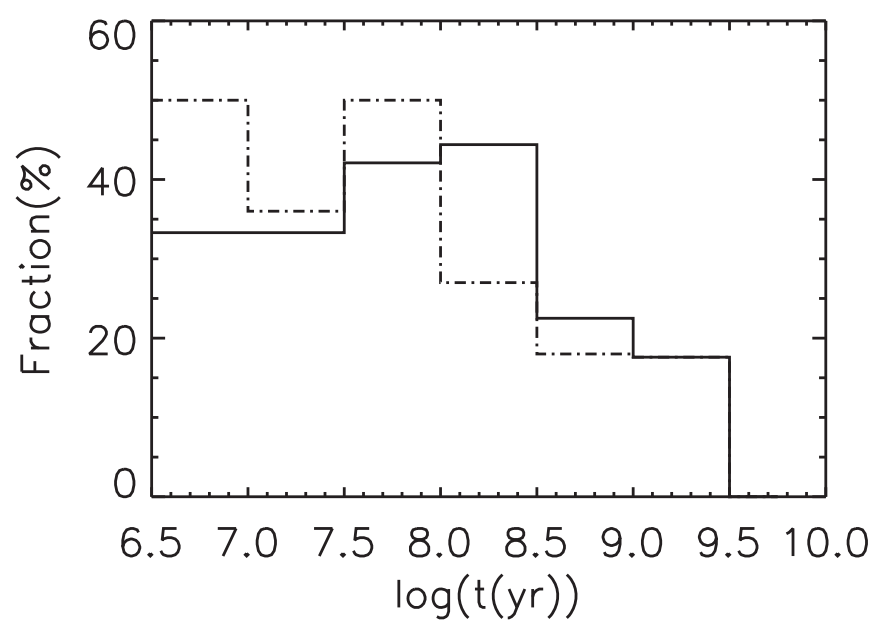

Figure 7. Fraction of clusters with Be star candidates. Solid lines: all clusters; dashed lines: clusters with distance $>1.5 \mathrm{kpc}$. Clusters with age $7.5<$ $\log (t$ (year $)) \leqslant 8.5$ tend to have Be star candidates. 


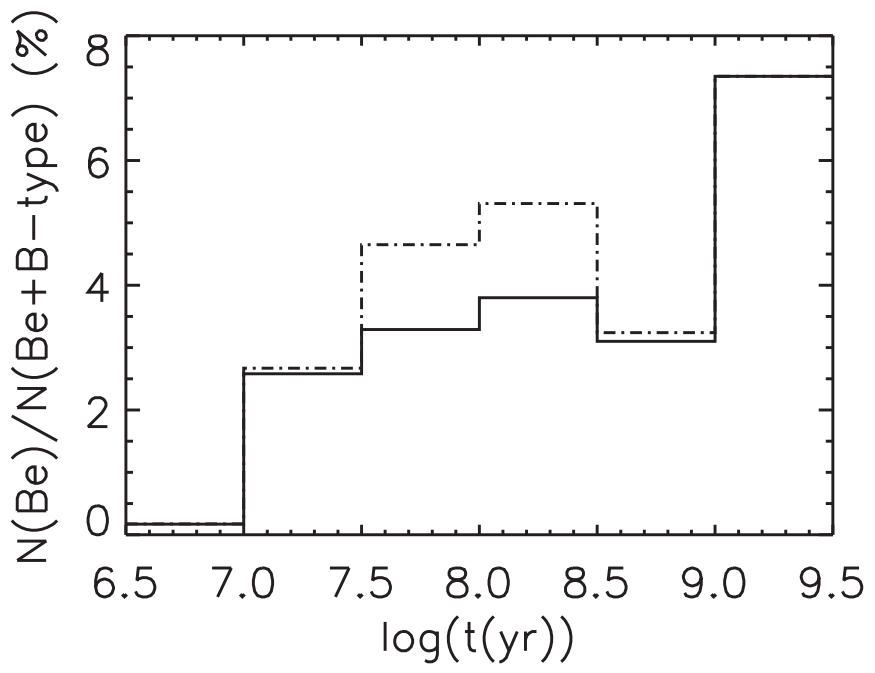

Figure 8. Be fraction $\mathrm{N}(\mathrm{Be}) / \mathrm{N}(\mathrm{Be}+\mathrm{B}$-type) of clusters with different ages. Solid lines: all clusters; dashed lines: clusters with distance $>1.5 \mathrm{kpc}$. Clusters within age bin of $7.5<\log (t$ (year $)) \leqslant 8.5$ tend to have a higher $\mathrm{Be}$ fraction $\mathrm{N}(\mathrm{Be}) / \mathrm{N}(\mathrm{Be}+\mathrm{B}$-type $)$.

$7.5<\log (t$ (year $)) \leqslant 8$ (dashed histogram in Figure 7$)$. Although it seems that clusters with the age of $6.5<\log (t($ year $)) \leqslant 7.0$ have higher value, we noted that this is caused by a small sample (only two clusters) in the bin. It is unlikely that the youngest clusters have a high probability of harboring Be stars, because they are mostly still in the pre-main sequence phase at this age.

\subsection{Be Star Fraction versus Age}

We compared the $\mathrm{Be}$ fraction $\mathrm{N}(\mathrm{Be}) / \mathrm{N}(\mathrm{Be}+\mathrm{B}$-type $)$ by summing all stars within the same age bin, e.g., each age bin shows a ratio of all $\mathrm{Be}$ star candidates to all B-types stars (Be +B-type). In Figure 8 (solid histogram), the ratio increases with ages and shows a peak at $8.0<\log (t$ (year $)) \leqslant 8.5$, then declines with older ages. As mentioned above, it is required to consider the clusters with distance $>1.5 \mathrm{kpc}$ to constrain the completeness. As shown in the dashed histogram in Figure 8, the fraction has a similar trend and the same peak as the original distribution. This result suggests that B-type stars with $7.5<\log (t$ (year $)) \leqslant 8.5$ tend to have more Be star candidates. On the other hand, it should be noted that the highest ratio seems to be in the oldest bin $9.0<\log (t$ (year) $) \leqslant 9.5$ at first glance. We examined those clusters older than $\log (t$ (year $))=9$ and found that the main contribution of Be and B-type stars in this age bin is from cluster Collinder 110; it has 7 Be candidates and $47 \mathrm{~B}$-type stars. If we remove this cluster, the Be fraction drops to $\sim 3 \%$, which is lower than that of clusters with $7.0<\log (t$ (year $)) \leqslant 8.5$. The overabundance of Be star candidates in Collinder 110 should be further confirmed using spectroscopy.

To avoid the effects of extreme numbers of individual clusters, we averaged the Be fraction of clusters within the same age bin. In Figure 9, we showed the average of Be frequency as a function of cluster ages. The age index with $8.0<\log (t$ (year $)) \leqslant 8.5$ exhibits the highest mean Be fraction, and the fraction decreases toward the older cluster ages (filled circles in Figure 9). If we consider the completeness, the clusters with $7.5<\log (t$ (year $)) \leqslant$ 9 have a high mean Be fraction (open diamond in Figure 9). For comparison, McSwain \& Gies (2005) found the peak among clusters with $7.4<\log (t$ (year $))<8$, and Fabregat \& Torrejón (2000) showed the peak is at $7.0<\log (t$ (year) $)<7.4$. The

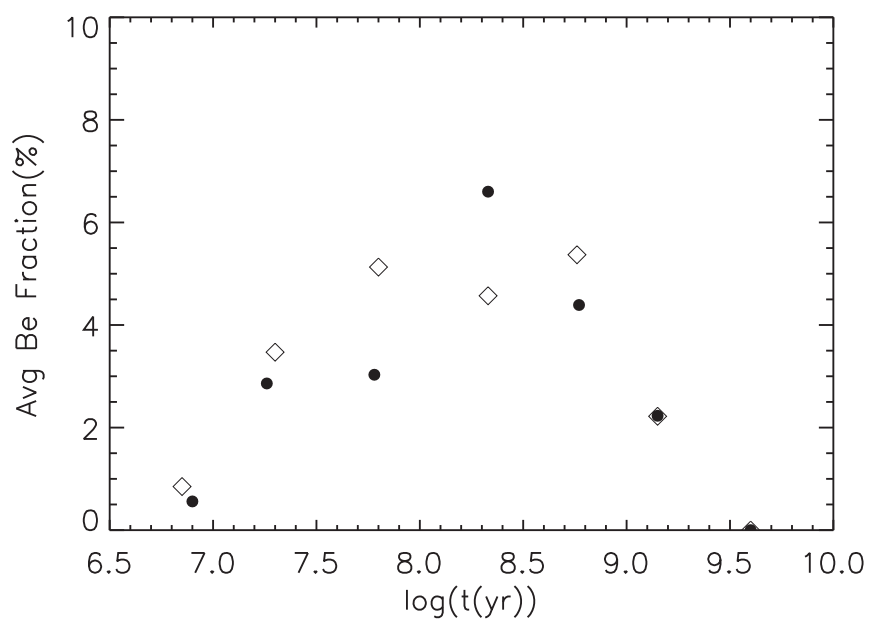

Figure 9. Averaged $\mathrm{Be}$ fraction $\mathrm{N}(\mathrm{Be}) / \mathrm{N}(\mathrm{Be}+\mathrm{B}$-type) of clusters with different ages. Filled circles: all clusters; open diamonds: clusters with distance $>1.5 \mathrm{kpc}$. The averaged Be fraction arises after the age of $\log (t($ year $))=7.5$ and then decreases after the age of $\log (t($ year $))=8.5$.

difference in these results is caused by different levels of completeness. Fabregat \& Torrejón (2000) and McSwain \& Gies (2005) have more complete early B-type stars, while we have a more complete sample of Be star candidates with spectral types later than B5.

\subsection{Concentration of Be Star Candidates}

The effects of mass segregation are essential in the dynamical evolution of open clusters, e.g., more massive stars are preferentially located in the central regions (Raboud \& Mermilliod 1998; Littlefair et al. 2003; Chen et al. 2007). The effects are suggested to be caused by kinetic energy redistribution and equipartition (Bonnell \& Davies 1998; de Grijs et al. 2002). Because Be stars are massive stars, the spatial distribution of Be stars in clusters with different ages might reveal the dynamical interaction snapshot among other members. Our cluster sample covers a wide range of ages from a few Myr to a few Gyr. We therefore investigate the concentration level with age by dividing the sample into young clusters $(7<\log (t$ (year $)) \leqslant 8)$ and intermediate/old clusters $(8<\log (t$ (year $) \leqslant 10)$. Due to the small number of old clusters, we combine the samples of intermediate and old clusters. For each cluster, we estimated the Be star fraction $\mathrm{N}(\mathrm{Be}) / \mathrm{N}(\mathrm{Be}+\mathrm{B}-$ type) within four annuli; each annulus has a width of $1 \sigma$ (Figure 10). The value $\sigma$ is determined by fitting the radial density profile (see Section 3 and Figure 3 ). Then we averaged the Be star fraction within the same annulus of all clusters within the same age bin. In Figure 11, we show the average Be star fractions within different annuli. From the point of view of the cluster age, the Be fraction within the second annulus of the intermediate and old clusters is slightly higher than that of the young clusters within the same annulus. On the other hand, the clusters with intermediate ages have similar Be fractions to those of the young clusters within the other three annuli. However, either in the young or intermediate/old clusters, the Be star candidates distribute uniformly. The results might indicate that Be star candidates do not form preferentially in the central regions of clusters. A larger sample of clusters is crucial to conduct a statistical study and to test this hypothesis in the future. 

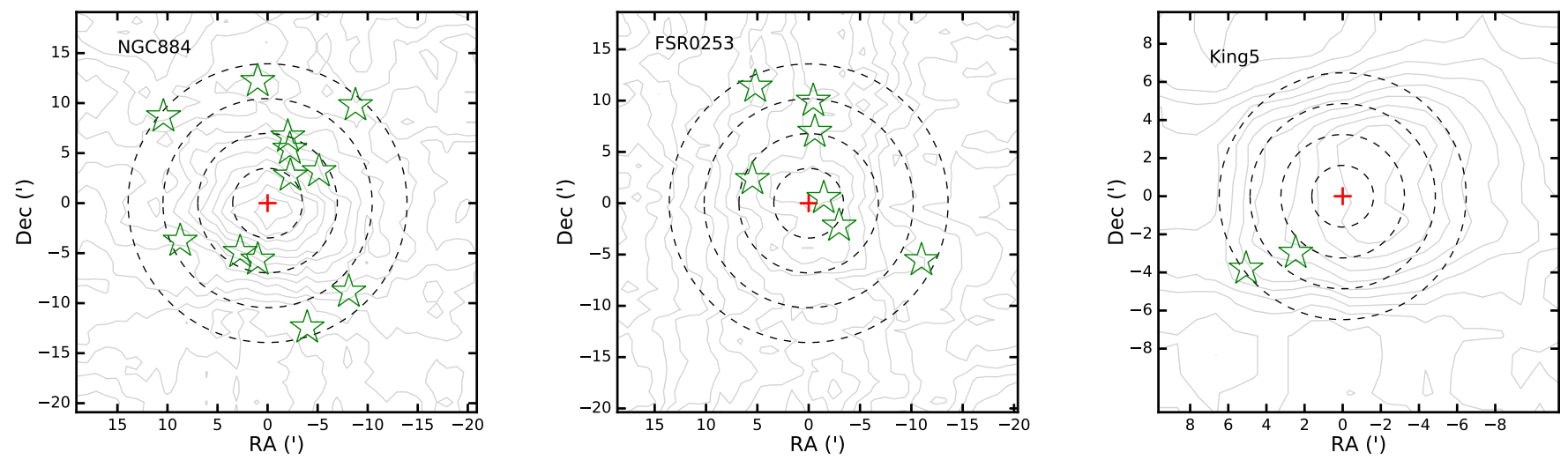

Figure 10. Examples of annulus definition for three clusters with different ages. Dashed circles represent the $1 \sigma, 2 \sigma, 3 \sigma$, and $4 \sigma$ radii for each cluster. Green pentagrams are Be candidates.

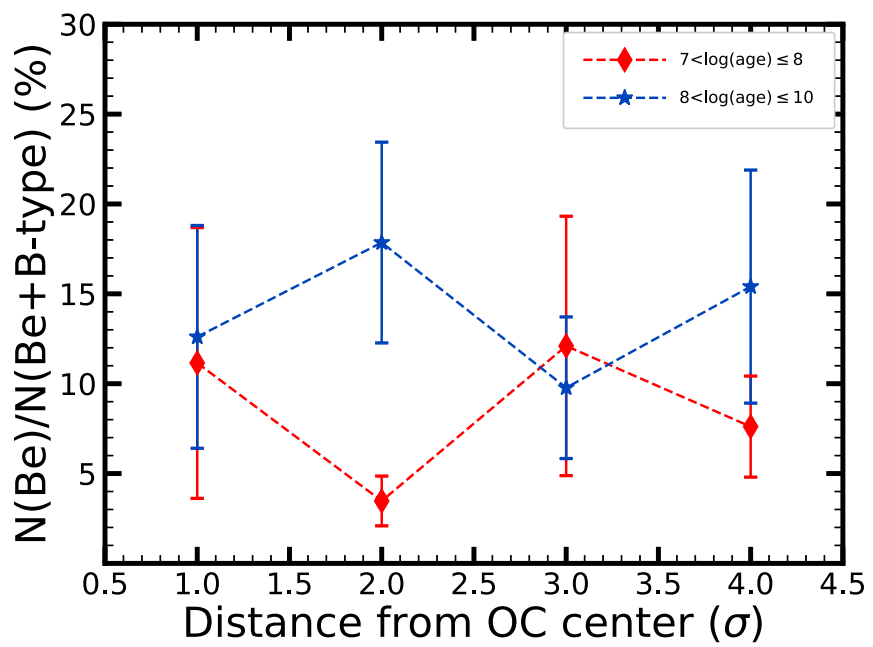

Figure 11. The concentration level of Be candidates. The lines with different colors show different age ranges. The Be fractions are the average ratios of $\mathrm{Be} /(\mathrm{Be}+\mathrm{B}$-type) within the same age bin and annulus. Spatial distribution of Be star candidates with different ages suggests that the distribution is ageindependent.

\subsection{Infrared Colors}

To further investigate infrared properties of Be candidates, we obtained the middle infrared (MIR) data from the Wide-field Infrared Survey Explorer (WISE; Wright et al. 2010) All-Sky Release. ${ }^{14}$ The WISE mission has mapped the entire sky in four bands at 3.4, 4.6, 12, and $22 \mu \mathrm{m}$ (hereafter called $W 1, W 2, W 3$, and W4) with resolutions of 6." 1 and 6." 5 for $W 1, W 2$ and $W 3$, and 12 ". 0 for $W 4$. All four bands were imaged simultaneously, and the exposure times were $7.7 \mathrm{~s}$ in $W 1$ and $W 2$ and $8.8 \mathrm{~s}$ in $W 3$ and $W 4$. The data presented here have been calibrated with the WISE pipeline.

We performed photometry for these candidates on the $W 1$, $W 2, W 3$, and $W 4$ images and measured their integrated brightness using the method described in Hsia \& Zhang (2014). After an initial inspection, we found that the detected $\mathrm{S} / \mathrm{N}$ at the $W 4$ band is very low for most candidates in our sample. We therefore limited our study to the objects in the searching range of the $W 1, W 2$, and $W 3$ bands and restricted our sources to have $\mathrm{S} / \mathrm{N}>4$ in all three bands. To estimate the uncertainties in brightness, we adopt the standard deviations of all background-subtracted brightness measurements. The

\footnotetext{
${ }^{14} \mathrm{http} / /$ wise2.ipac.caltech.edu/docs/release/allsky/
}

characteristic uncertainties of all brightness measurements are estimated to be $\sim 6 \%, 5 \%$, and $21 \%$ for $W 1, W 2$, and $W 3$ bands, respectively. In total, $61 \mathrm{Be}$ star candidates have $W 1, W 2$, and $W 3$ band data with sufficient $\mathrm{S} / \mathrm{N}$ to study. A summary of WISE photometric results for the sources is given in Table 3, and their corresponding $W 1-W 2$ and $W 2-W 3$ color-color diagrams are shown in Figures 12 and 13.

In Figure 12, we compare their $W 1-W 2$ and $W 2-W 3$ colors with B type stars (Paunzen et al. 2005) and known Be stars (Zhang et al. 2005). More than $80 \%$ of our Be candidates are located around the upper-left of this color diagram. They also show similar color distributions as known Be stars, which is suggested to be caused by free-free emission or bound-free emission from proton-electron scattering (Chen et al. 2016). On the other hand, as noted by Chen et al. (2016), some Be stars located at the lower-right region might be indicative of the presence of circumstellar dust. However, none of our Be candidates are located in the lower-right region, suggesting their infrared excess are mainly due to free-free emission or bound-free emission.

The infrared color excess represents variations of circumstellar plasma or contribution from the ionized material. Dougherty et al. (1991) found that early type Be stars have larger IR color excesses than late type ones do, which are attributed to either dust emission or effect of photospheric emission by cool circumstellar disk. However, no correlation between WISE color and spectral type was found suggested by Chen et al. (2016).

To examine their age dependence, we plotted the $W 1-W 2$ versus $W 2-W 3$ diagram on the basis of their cluster ages. As shown in Figure 13, clearly, mid-infrared color excess are independent of the ages, indicating that the circumstellar material might not be correlated with their ages. Previous works also show relevant results about age independence of performance mainly in near-infrared, despite lack of sample clusters in different age. Wisniewski et al. (2007) presented imaging polarization observations of Be stars in the clusters of Magellanic Clouds. The Be stars in the youngest clusters show a really low IR color excess and distribute evenly as well as other candidates in the older clusters. On the other hand, a Be star with large IR excess still can be found in an older cluster of Magellanic Clouds reported by Paul et al. (2017). These works including ours indicated that NIR and MIR colors are not likely correlated with age, implying that the disk forming mechanisms, such as rapid rotation and pulsation, are not age dependent. 
Table 3

WISE Photometric Data of Be Candidates

\begin{tabular}{|c|c|c|c|c|c|c|c|c|c|}
\hline Object & $\begin{array}{c}3.4 \mu \mathrm{m} \\
(\mathrm{mag})\end{array}$ & $\begin{array}{c}\sigma_{3.4 \mu \mathrm{m}} \\
(\mathrm{mag})\end{array}$ & $\begin{array}{c}4.6 \mu \mathrm{m} \\
(\mathrm{mag})\end{array}$ & $\begin{array}{r}\sigma_{4.6 \mu \mathrm{m}} \\
(\mathrm{mag})\end{array}$ & $\begin{array}{l}12 \mu \mathrm{m} \\
(\mathrm{mag})\end{array}$ & $\begin{array}{l}\sigma_{12 \mu \mathrm{m}} \\
(\mathrm{mag})\end{array}$ & $\log (t($ year $))$ & $\begin{array}{c}W 1-W 2 \\
(\mathrm{mag})\end{array}$ & $\begin{array}{c}W 2-W 3 \\
\text { (mag) }\end{array}$ \\
\hline Stock 20-1 & 10.708 & 0.023 & 10.733 & 0.021 & 10.425 & 0.122 & 8.337 & -0.025 & 0.308 \\
\hline Stock 21-1 & 11.605 & 0.021 & 11.686 & 0.021 & 11.407 & 0.112 & 8.825 & -0.081 & 0.279 \\
\hline Stock 21-2 & 12.288 & 0.023 & 12.309 & 0.023 & 12.006 & 0.173 & 8.825 & -0.021 & 0.303 \\
\hline Stock 21-3 & 11.892 & 0.023 & 11.897 & 0.022 & 11.685 & 0.148 & 8.825 & -0.005 & 0.212 \\
\hline NGC 743-1 & 9.174 & 0.022 & 8.822 & 0.021 & 7.901 & 0.019 & 8.290 & 0.352 & 0.921 \\
\hline NGC 743-2 & 10.988 & 0.023 & 11.015 & 0.020 & 11.125 & 0.127 & 8.290 & -0.027 & -0.110 \\
\hline NGC 743-3 & 10.972 & 0.023 & 10.993 & 0.021 & 11.154 & 0.125 & 8.290 & -0.021 & -0.161 \\
\hline NGC 743-4 & 10.783 & 0.023 & 10.657 & 0.021 & 10.041 & 0.064 & 8.290 & 0.126 & 0.616 \\
\hline NGC 744-1 & 10.934 & 0.023 & 10.961 & 0.022 & 10.990 & 0.127 & 8.375 & -0.027 & -0.029 \\
\hline NGC 744-5 & 11.538 & 0.022 & 11.565 & 0.022 & 11.688 & 0.252 & 8.375 & -0.027 & -0.123 \\
\hline NGC 744-13 & 9.958 & 0.023 & 9.961 & 0.021 & 9.861 & 0.045 & 8.375 & -0.003 & 0.100 \\
\hline NGC 869-1 & 11.166 & 0.024 & 11.184 & 0.021 & 11.071 & 0.123 & 7.280 & -0.018 & 0.113 \\
\hline NGC 869-2 & 11.071 & 0.024 & 11.095 & 0.021 & 11.503 & 0.175 & 7.280 & -0.024 & -0.408 \\
\hline NGC 869-3 & 12.225 & 0.024 & 12.276 & 0.023 & $\ldots$ & $\ldots$ & 7.280 & -0.051 & $\ldots$ \\
\hline NGC 869-4 & 12.852 & 0.025 & 12.871 & 0.024 & $\ldots$ & $\ldots$ & 7.280 & -0.019 & $\ldots$ \\
\hline NGC 869-14 & 10.754 & 0.023 & 10.780 & 0.021 & 10.873 & 0.108 & 7.280 & -0.026 & -0.093 \\
\hline NGC 869-15 & 10.831 & 0.022 & 10.855 & 0.022 & 11.002 & 0.109 & 7.280 & -0.024 & -0.147 \\
\hline NGC 869-16 & 11.024 & 0.023 & 11.035 & 0.020 & 11.028 & 0.116 & 7.280 & -0.011 & 0.007 \\
\hline NGC 869-17 & 11.396 & 0.024 & 11.439 & 0.021 & $\ldots$ & $\cdots$ & 7.280 & -0.043 & $\cdots$ \\
\hline NGC 869-18 & 12.112 & 0.023 & 12.123 & 0.023 & $\ldots$ & $\ldots$ & 7.280 & -0.011 & $\ldots$ \\
\hline NGC 869-19 & 12.675 & 0.024 & 12.691 & 0.025 & $\ldots$ & $\ldots$ & 7.280 & -0.016 & $\ldots$ \\
\hline NGC 869-20 & 12.943 & 0.024 & 12.969 & 0.025 & $\ldots$ & $\ldots$ & 7.280 & -0.026 & $\ldots$ \\
\hline NGC 869-25 & 11.842 & 0.082 & 11.856 & 0.038 & $\ldots$ & $\ldots$ & 7.280 & -0.014 & $\ldots$ \\
\hline NGC 869-26 & 10.281 & 0.071 & 10.008 & 0.021 & 9.073 & 0.030 & 7.280 & 0.273 & 0.935 \\
\hline NGC 869-27 & 10.512 & 0.023 & 10.255 & 0.020 & 9.395 & 0.034 & 7.280 & 0.257 & 0.860 \\
\hline Basel 10-1 & 9.807 & 0.023 & 9.534 & 0.021 & 8.780 & 0.029 & 7.600 & 0.273 & 0.754 \\
\hline NGC 886-1 & 10.455 & 0.024 & 10.171 & 0.020 & 9.188 & 0.034 & 8.750 & 0.284 & 0.983 \\
\hline NGC 884-1 & 10.150 & 0.023 & 10.176 & 0.020 & 9.809 & 0.045 & 7.200 & -0.026 & 0.367 \\
\hline NGC $884-2$ & 10.405 & 0.023 & 10.405 & 0.021 & 10.416 & 0.087 & 7.200 & 0.000 & -0.011 \\
\hline NGC 884-3 & 11.446 & 0.023 & 11.453 & 0.023 & 11.350 & 0.150 & 7.200 & -0.007 & 0.103 \\
\hline NGC 884-4 & 11.518 & 0.024 & 11.577 & 0.026 & $\ldots$ & $\ldots$ & 7.200 & -0.059 & $\cdots$ \\
\hline NGC 884-5 & 11.381 & 0.024 & 11.400 & 0.021 & 11.390 & 0.143 & 7.200 & -0.019 & 0.010 \\
\hline NGC 884-6 & 12.460 & 0.026 & 12.511 & 0.025 & $\ldots$ & $\ldots$ & 7.200 & -0.051 & $\ldots$ \\
\hline NGC 884-8 & 11.169 & 0.024 & 11.255 & 0.023 & $\ldots$ & $\ldots$ & 7.200 & -0.086 & $\ldots$ \\
\hline NGC 884-11 & 11.694 & 0.023 & 11.703 & 0.022 & 11.443 & 0.195 & 7.200 & -0.009 & 0.260 \\
\hline NGC 884-12 & 12.000 & 0.128 & 12.134 & 0.022 & 10.343 & 0.091 & 7.200 & -0.134 & 1.791 \\
\hline NGC 884-13 & 13.081 & 0.025 & 13.147 & 0.031 & $\ldots$ & $\ldots$ & 7.200 & -0.066 & $\ldots$ \\
\hline NGC 884-14 & 12.675 & 0.024 & 12.691 & 0.025 & $\ldots$ & $\ldots$ & 7.200 & -0.016 & $\ldots$ \\
\hline NGC 884-19 & 10.512 & 0.023 & 10.255 & 0.020 & 9.395 & 0.034 & 7.200 & 0.257 & 0.860 \\
\hline NGC 957-1 & 9.974 & 0.023 & 9.783 & 0.020 & 9.412 & 0.036 & 7.250 & 0.191 & 0.371 \\
\hline King 5-1 & 12.441 & 0.023 & 12.452 & 0.024 & $\ldots$ & $\ldots$ & 9.090 & -0.011 & $\ldots$ \\
\hline King 5-2 & 12.228 & 0.023 & 12.215 & 0.024 & $\cdots$ & $\cdots$ & 9.090 & 0.013 & $\ldots$ \\
\hline NGC 1513-1 & 11.002 & 0.023 & 11.011 & 0.021 & 10.883 & 0.111 & 8.500 & -0.009 & 0.128 \\
\hline NGC 1513-2 & 11.511 & 0.022 & 11.535 & 0.021 & $\ldots$ & $\ldots$ & 8.500 & -0.024 & $\ldots$ \\
\hline NGC 1513-3 & 11.647 & 0.023 & 11.626 & 0.023 & 11.711 & 0.240 & 8.500 & 0.021 & -0.085 \\
\hline Berkeley 1-1 & 12.081 & 0.024 & 12.073 & 0.023 & 11.718 & 0.253 & 8.700 & 0.008 & 0.355 \\
\hline NGC 1664-1 & 11.541 & 0.023 & 11.547 & 0.021 & 11.739 & 0.248 & 8.750 & -0.006 & -0.192 \\
\hline NGC 1778-1 & 10.419 & 0.023 & 10.317 & 0.020 & 9.715 & 0.050 & 8.450 & 0.102 & 0.602 \\
\hline FSR 0749-1 & 12.168 & 0.023 & 12.173 & 0.024 & $\ldots$ & $\ldots$ & 8.620 & -0.005 & $\ldots$ \\
\hline FSR 0749-2 & 11.850 & 0.023 & 11.872 & 0.022 & $\ldots$ & $\ldots$ & 8.620 & -0.022 & $\ldots$ \\
\hline FSR 0799-1 & 12.465 & 0.024 & 12.367 & 0.025 & 11.711 & 0.259 & 9.095 & 0.098 & 0.656 \\
\hline FSR 0816-1 & 12.575 & 0.025 & 12.574 & 0.027 & $\ldots$ & $\ldots$ & 7.800 & 0.001 & $\ldots$ \\
\hline FSR 0904-1 & 10.581 & 0.024 & 10.322 & 0.021 & 9.552 & 0.058 & 7.800 & 0.259 & 0.770 \\
\hline Kronberger 12-1 & 11.825 & 0.023 & 11.803 & 0.025 & $\cdots$ & $\cdots$ & 8.870 & 0.022 & $\cdots$ \\
\hline FSR 0902-1 & 11.381 & 0.022 & 11.409 & 0.022 & 10.723 & 0.251 & 7.145 & -0.028 & 0.686 \\
\hline Skiff J0619+18.5-1 & 11.277 & 0.024 & 11.316 & 0.022 & 10.677 & 0.110 & 7.900 & -0.039 & 0.639 \\
\hline Skiff J0619+18.5-2 & 10.957 & 0.026 & 10.988 & 0.024 & 10.984 & 0.200 & 7.900 & -0.031 & 0.004 \\
\hline Skiff J0619+18.5-3 & 11.159 & 0.024 & 11.174 & 0.022 & 11.313 & 0.181 & 7.900 & -0.015 & -0.139 \\
\hline Skiff J0619+18.5-4 & 11.495 & 0.023 & 11.483 & 0.022 & 11.400 & 0.187 & 7.900 & 0.012 & 0.083 \\
\hline Skiff J0619+18.5-5 & 11.403 & 0.024 & 11.382 & 0.022 & 11.338 & 0.223 & 7.900 & 0.021 & 0.044 \\
\hline Skiff J0619+18.5-11 & 11.223 & 0.023 & 11.240 & 0.022 & 11.140 & 0.139 & 7.900 & -0.017 & 0.100 \\
\hline Skiff J0619+18.5-12 & 11.682 & 0.024 & 11.708 & 0.023 & $\cdots$ & $\cdots$ & 7.900 & -0.026 & $\cdots$ \\
\hline Skiff J0619+18.5-13 & 11.818 & 0.023 & 11.813 & 0.023 & $\cdots$ & $\cdots$ & 7.900 & 0.005 & $\ldots$ \\
\hline
\end{tabular}


Table 3

(Continued)

\begin{tabular}{|c|c|c|c|c|c|c|c|c|c|}
\hline Object & $\begin{array}{c}3.4 \mu \mathrm{m} \\
(\mathrm{mag})\end{array}$ & $\begin{array}{c}\sigma_{3.4 \mu \mathrm{m}} \\
(\mathrm{mag})\end{array}$ & $\begin{array}{c}4.6 \mu \mathrm{m} \\
(\mathrm{mag})\end{array}$ & $\begin{array}{c}\sigma_{4.6 \mu \mathrm{m}} \\
(\mathrm{mag})\end{array}$ & $\begin{array}{c}12 \mu \mathrm{m} \\
(\mathrm{mag})\end{array}$ & $\begin{array}{l}\sigma_{12 \mu \mathrm{m}} \\
(\mathrm{mag})\end{array}$ & $\log (t$ (year $))$ & $\begin{array}{c}W 1-W 2 \\
(\mathrm{mag})\end{array}$ & $\begin{array}{c}W 2-W 3 \\
\text { (mag) }\end{array}$ \\
\hline Dolidze 22-1 & 12.407 & 0.023 & 12.441 & 0.023 & $\cdots$ & $\cdots$ & 8.370 & -0.034 & $\cdots$ \\
\hline FSR 0955-1 & 10.275 & 0.024 & 10.300 & 0.020 & 10.265 & 0.092 & 7.400 & -0.025 & 0.035 \\
\hline NGC 2244-1 & 11.183 & 0.025 & 11.225 & 0.024 & $\cdots$ & $\cdots$ & 6.700 & -0.042 & $\cdots$ \\
\hline FSR 0905-1 & 11.261 & 0.024 & 11.289 & 0.022 & $\cdots$ & $\cdots$ & 8.300 & -0.028 & $\cdots$ \\
\hline FSR 0905-2 & 12.239 & 0.024 & 12.206 & 0.029 & $\ldots$ & $\ldots$ & 8.300 & 0.033 & $\ldots$ \\
\hline Basel 8-2 & 10.691 & 0.022 & 10.716 & 0.021 & 11.214 & 0.259 & 7.925 & -0.025 & -0.498 \\
\hline Basel 8-3 & 11.875 & 0.023 & 11.927 & 0.023 & $\cdots$ & $\cdots$ & 7.925 & -0.052 & $\cdots$ \\
\hline Collinder 110-1 & 12.212 & 0.024 & 12.202 & 0.025 & $\cdots$ & $\cdots$ & 9.220 & 0.010 & $\cdots$ \\
\hline Collinder 110-2 & 12.929 & 0.024 & 12.957 & 0.028 & $\cdots$ & $\cdots$ & 9.220 & -0.028 & $\cdots$ \\
\hline Collinder 110-3 & 13.068 & 0.026 & 13.066 & 0.033 & $\cdots$ & $\cdots$ & 9.220 & 0.002 & $\cdots$ \\
\hline Collinder 110-4 & 12.342 & 0.024 & 12.324 & 0.023 & $\cdots$ & $\cdots$ & 9.220 & 0.018 & $\cdots$ \\
\hline NGC 6866-2 & 11.506 & 0.023 & 11.552 & 0.021 & 11.785 & 0.224 & 8.640 & -0.046 & -0.233 \\
\hline NGC 6866-3 & 11.332 & 0.023 & 11.381 & 0.021 & 11.905 & 0.218 & 8.640 & -0.049 & -0.524 \\
\hline NGC 6866-12 & 11.781 & 0.023 & 11.810 & 0.022 & 11.125 & 0.127 & 8.640 & -0.029 & 0.685 \\
\hline NGC 6910-1 & 10.900 & 0.023 & 10.903 & 0.020 & 9.967 & 0.109 & 7.530 & -0.003 & 0.936 \\
\hline FSR 0253-1 & 10.235 & 0.022 & 10.274 & 0.020 & 10.266 & 0.062 & 8.275 & -0.039 & 0.008 \\
\hline FSR 0253-2 & 10.689 & 0.023 & 10.707 & 0.020 & 10.348 & 0.054 & 8.275 & -0.018 & 0.359 \\
\hline FSR 0253-3 & 11.611 & 0.023 & 11.640 & 0.021 & 11.313 & 0.140 & 8.275 & -0.029 & 0.327 \\
\hline FSR 0253-4 & 11.952 & 0.023 & 11.965 & 0.022 & 12.061 & 0.224 & 8.275 & -0.013 & -0.096 \\
\hline FSR 0253-5 & 11.770 & 0.023 & 11.770 & 0.021 & 11.891 & 0.216 & 8.275 & 0.000 & -0.121 \\
\hline FSR 0253-6 & 11.831 & 0.023 & 11.875 & 0.021 & 12.020 & 0.246 & 8.275 & -0.044 & -0.145 \\
\hline FSR 0253-7 & 11.528 & 0.023 & 11.505 & 0.021 & 11.905 & 0.184 & 8.275 & 0.023 & -0.400 \\
\hline FSR 0354-1 & 9.364 & 0.023 & 9.062 & 0.020 & 8.111 & 0.021 & 8.000 & 0.302 & 0.951 \\
\hline FSR 0354-2 & 9.300 & 0.022 & 8.895 & 0.019 & 7.948 & 0.018 & 8.000 & 0.405 & 0.947 \\
\hline
\end{tabular}

Note. Column 1: Names of Be candidates. Column 2: Magnitudes of the $W 1$ band. Column 3: Uncertainties of the $W 1$ band. Column 4: Magnitudes of the $W 2$ band Column 5: Uncertainties of the $W 2$ band. Column 6: Magnitudes of the $W 3$ band. Column 7: Uncertainties of the W3 band. Column 8: Ages of host clusters. Column 9: W1-W2. Column 10: W2-W3.

\subsection{Optical Variability}

Be stars exhibit photometric and spectroscopic variability with various timescales of days to years (Hubert et al. 1997; Hubert \& Floquet 1998; Percy et al. 2002; Kourniotis et al. 2014; Labadie-Bartz et al. 2017). It has been suggested that Be stars have different phases of the circumstellar disk, such as ejecting material from the stellar surface, disk growth, and dissipation, which could cause variability. While outbursts or variability on short and intermediate timescales of days to months are believed to be caused by non-radial pulsation, ejected material or stellar winds, the variability with long timescales of years to decades is associated with the appearance and disappearance of the disk or the global density oscillations (Okazaki 1997; Labadie-Bartz et al. 2017). Therefore, investigating long-term variability of Be stars in different environments could provide us with insight into their disk evolution. Investigating short-term and mid-term variability requires the survey with high cadences of hours and days. The current catalogs, with lower cadences of days to weeks or months, are more beneficial for studying long-term variability. Thus, we study long-term variability ( $>200-300$ days) for our Be star candidates in this work.
Time windows of the All Sky Automated Survey (ASAS ${ }^{15}$; Pojmanski 1997) and PTF/iPTF allow us to investigate the long-term variability of Be star candidates. We adopted the ASAS data to investigate the variability for Be star candidates with $R$-band magnitudes brighter than 14 . The ASAS surveyed the available sky south of decl. $+28^{\circ}$ using $I$ (ASAS-2, from 1998 to 2000) and $V$ (ASAS-3, from 2000 to 2009) band filters with a $8.5 \times 8.5$ field of view. We used ASAS- 3 database ${ }^{16}$ to search for the $V$-band variability of Be star candidates (see also Pojmański 2001). We used the search radius of $5^{\prime \prime}$ and only selected the data that is marked with "A" in the grade flag of the catalog, indicating the best data, to ensure that the photometry is convincing. Twenty-one of our Be candidates can be found in the ASAS-3 catalog.

On the other hand, PTF $R$-band data were adopted to investigate the variability of $\mathrm{Be}$ star candidates with $R$-band fainter than $14 \mathrm{mag}$. We used the search radius of $3^{\prime \prime}$ and selected observations with the "ipac" flag marked with 0 and with the "SEXtractor" flag $\leqslant 2$ in the catalog to ensure that the data qualify as reliable. We also neglected the sources that only

\footnotetext{
15 http://www.astrouw.edu.pl/asas/

16 http://www.astrouw.edu.pl/asas $/$ ?page $=$ aasc $\&$ catsrc $=$ asas3
} 


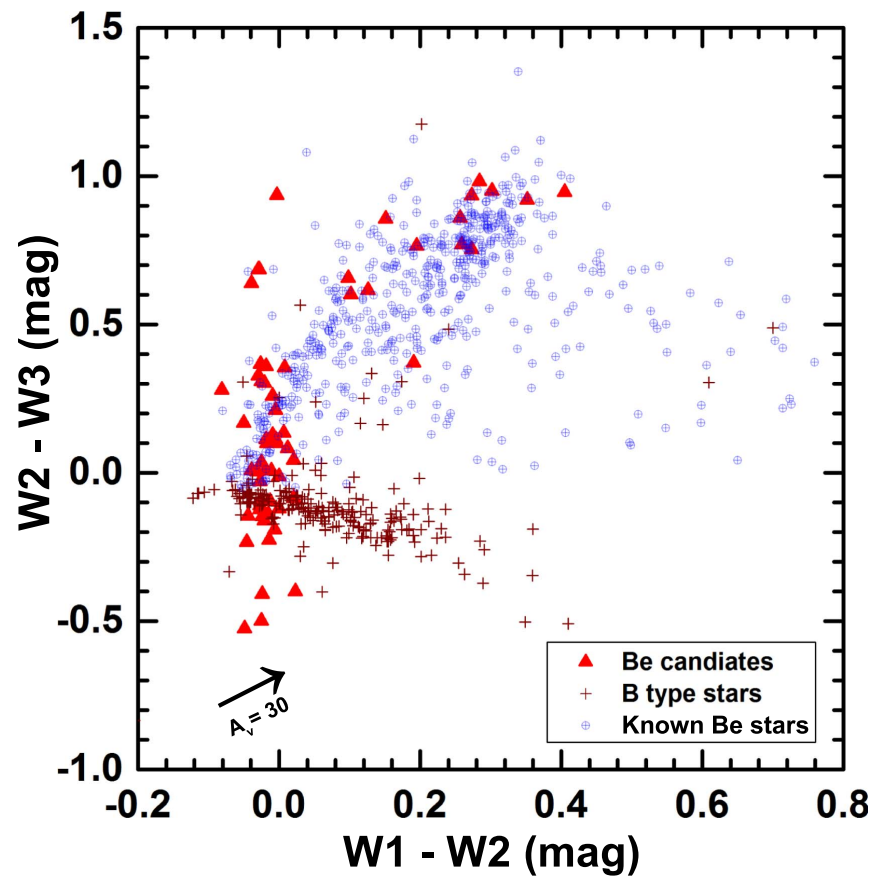

Figure 12. WISE $W 1-W 2$ vs. $W 2-W 3$ color-color diagram for studied cluster Be star candidates. Also plotted are 224 normal B type stars (brown crosses; Paunzen et al. 2005) and 538 known Be stars (blue circles; Zhang et al. 2005) with these Be star candidates of our sample (red triangles). Note that the MIR distribution of Be star candidates in our sample is similar to that of known Be stars.

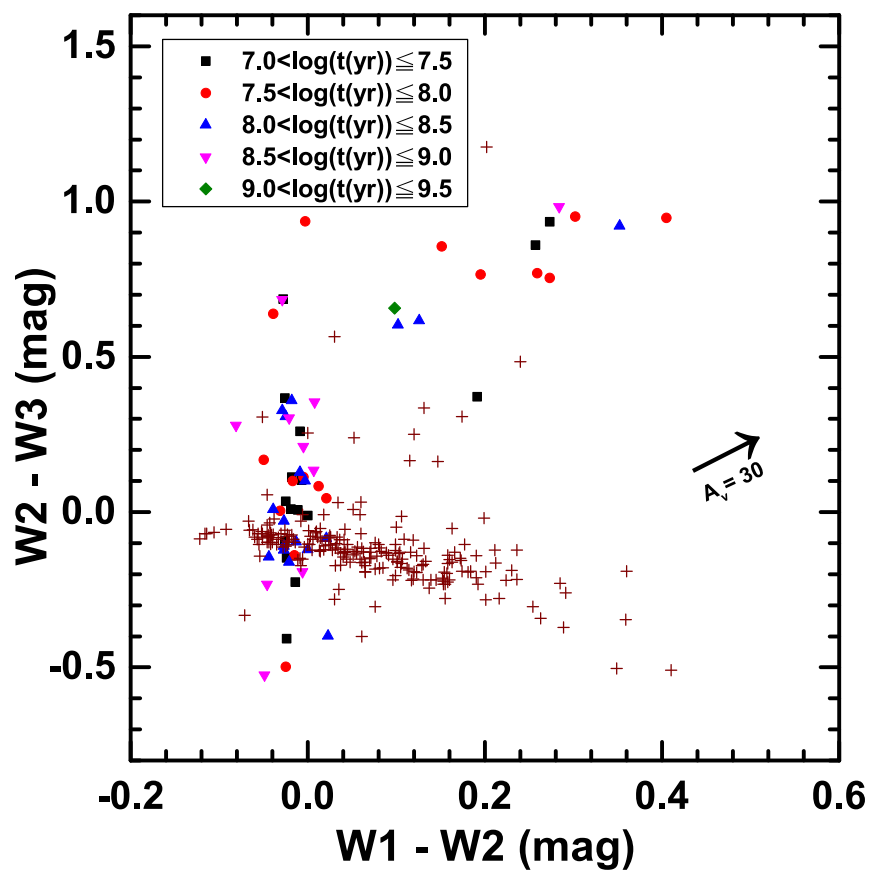

Figure 13. $W I S E W 1-W 2$ vs. $W 2-W 3$ color-color diagram for studied Be star candidates with different ages (blue and pink triangles, black squares, red circles, and green diamonds) plus normal B type stars (brown crosses; Paunzen et al. 2005) for comparison. The distributions of our Be star candidates are not related to their ages.

have data points less than 10 observations. In total, $10 \mathrm{Be}$ star candidates are selected from the PTF catalogue.

By applying differential photometry, among 31 Be star candidates with qualified photometric data points, we found that no Be star candidates have long-term variability within a

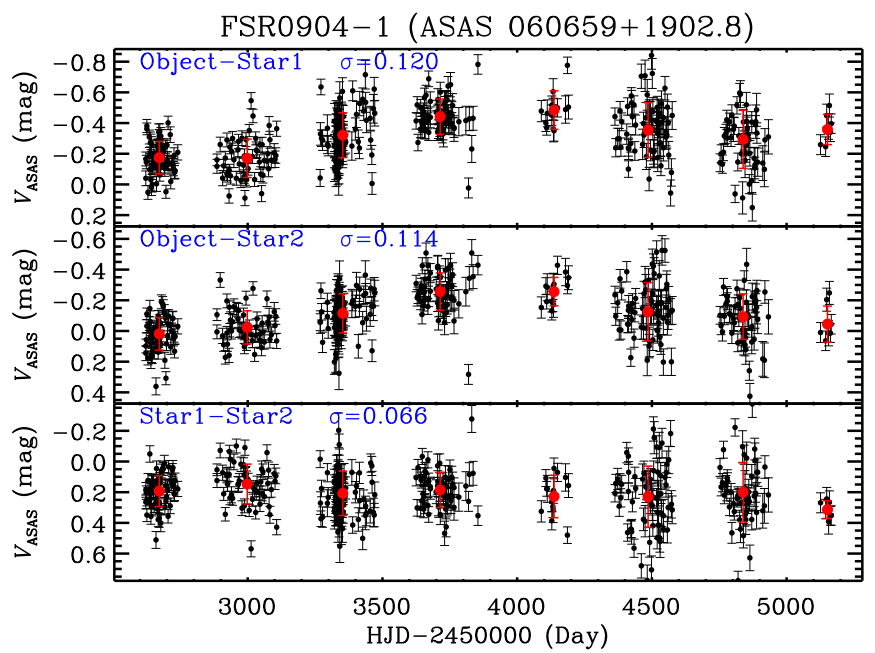

Figure 14. Differential photometry of the Be star candidate FSR 0904-1 and its comparison stars. The upper and middle panels show the differential light curves between FSR 0904-1 and its two comparison stars, ASAS 060659 +1902.8 and ASAS 060649+1901.2, respectively. The lower panel shows the differential light curve between the two comparison stars. The error-weighted mean of each epoch is represented by red dot. FSR 0904-1 exhibits greater variation $(\sigma=0.120$ and 0.114$)$ than its comparison stars $(\sigma=0.066)$.

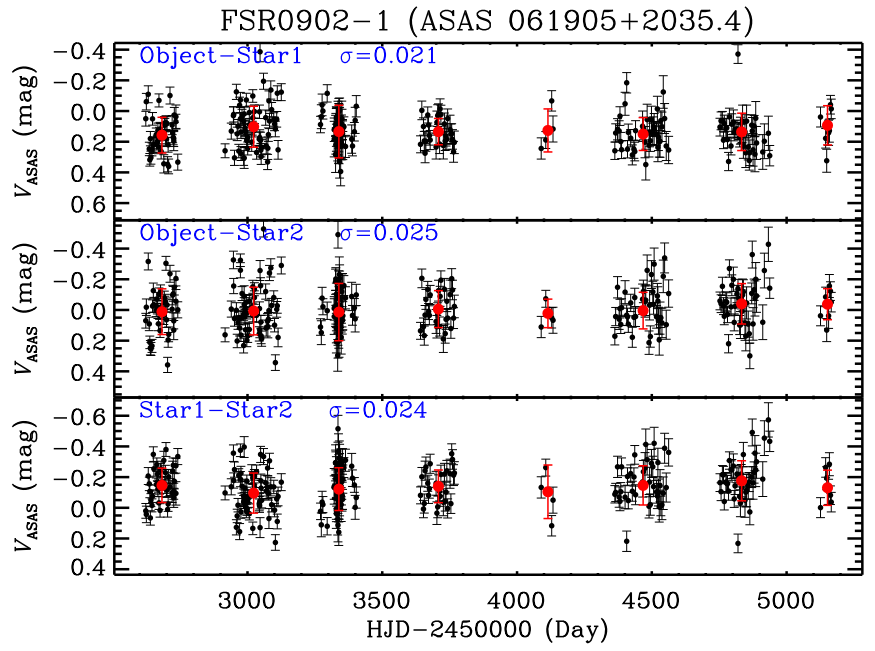

Figure 15. Differential photometry of the Be star candidate FSR 0902-1 and its comparison stars ASAS 061829+2025.9 and ASAS 061917+2038.8. FSR 0902-1 shows similar variation with its comparison stars, indicating that it has no variability.

timescale of several hundred days. However, we discovered that one Be candidate FSR 0904-1 shows prominent long-term variability, with an amplitude of $0.2-0.3 \mathrm{mag}$ on a longer timescale of $\sim 2000$ days. The differential photometry among the object FSR 0904-1 and two nearby comparison stars with similar brightness are shown in Figure 14. We estimated the mean photometric scatters of different epochs; while the $\mathrm{Be}$ candidate FSR 0904-1 has larger variation $(\sigma=0.120$ and $0.114)$, comparison stars show smaller variations $(\sigma=0.066)$, suggesting the Be candidate FSR 0904-1 has possible longterm variability. For comparison, the differential light curve of Be candidate FSR 0902-1, with no obvious long-term variability, is shown in Figure 15.

Mennickent et al. (2002) found that Be star candidates have four types of light curves. Type-1 stars show sharp or humplike outbursts with a mean timescale of 126 days that could be 
similar to the outbursting reported by Hubert \& Floquet (1998). Type-2 stars have sudden brightness increasing with an amplitude of a few tenths of magnitude, and remain a similar brightness for hundreds of days. High and low states are usually observed in type- 2 Be star candidates. Type- 3 Be star candidates show periodic or quasi-period oscillations, and type4 stars exhibit random variability on timescales of days to years. Sabogal et al. (2014) conducted a search for southern Galactic Be star candidates within the ASAS catalog; they found that only $9 \%$ of Be star candidates show outburst (Type-1 stars) while $91 \%$ of the candidates show random variations (Type-4 stars). Nevertheless, none of the Be star candidates reported by these studies show the similar long-term variations of FSR 0904-1.

Several possibilities might cause these kind of long-term variations. One possibility is the contamination of nearby stars due to poor angular resolution $\left(\sim 14^{\prime \prime}\right)$ of the ASAS survey. However, after examining PTF $R$-band images, we have ruled out this possibility. No nearby stars are located at the $15^{\prime \prime} \times 15^{\prime \prime}$ region of the Be candidate FSR 0904-1. The other possibility is a long-lived strong outburst. The timescale of a long-term outburst, however, is usually 200-300 days (Hubert $\&$ Floquet 1998). The long timescale of FSR 0904-1 variation is unlikely related to an outburst. Another possibility is the global density oscillations in the circumstellar disk (Haubois et al. 2012). As shown in Labadie-Bartz et al. (2017), they found that some Be stars also have similar long-term variability, on the timescale of 2000-3000 days. Using simultaneous spectroscopic observations, they suggested that the global density oscillations could cause the long-term variability observed in brightness change and violet-to red ratio $(\mathrm{V} / \mathrm{R})$ of the $\mathrm{H} \alpha$ line. Unfortunately, we are unable to test this hypothesis without simultaneous time-series spectroscopic measurements. However, the photometric variability usually has a positive relation with the variability of the $\mathrm{H} \alpha$ emissionline strength, which originated from the circumstellar disk. Thus, the long-term timescale of photometric variability also implies that the timescale of the disk evolution might be as long as $\sim 2000$ days.

\section{Summary and Conclusions}

We identified 96 Be star candidates in about $31 \%$ of the clusters (32/104). The clusters with a high Be fraction $\mathrm{N}(\mathrm{Be}) /$ $\mathrm{N}(\mathrm{Be}+\mathrm{B}$-type $)>20 \%$ tend to be located in spiral arms, which could indicate active star formation. More than half of the clusters with Be star candidates have Be fraction $\mathrm{N}(\mathrm{Be}) / \mathrm{N}(\mathrm{Be}$ + B-type $)<10 \%$, which is similar to previous studies. We found that clusters with age $7.5<\log (t$ (year $)) \leqslant 8.5$ tend to have Be star candidates. Also, clusters with age $7.5<$ $\log (t($ year $)) \leqslant 8.5$ have higher $\mathrm{Be}$ fraction $\mathrm{N}(\mathrm{Be}) / \mathrm{N}(\mathrm{Be}+\mathrm{B}-$ type). These results suggest that environments of young ( $\sim 30 \mathrm{Myr})$ and intermediate $(\sim 300 \mathrm{Myr})$ clusters could be beneficial to the formation of Be stars. We compared the concentration level of $\mathrm{Be}$ star candidates with different ages. We found that the Be star candidates in either young or old clusters are not centrally concentrated in the clusters. This implies that Be stars do not form preferentially in the central regions of clusters. We showed that Be star candidates have similar MIR colors to the known Be stars, and their MIR color excesses are independent of the ages. If the MIR color excesses are mainly due to free-free or bound-free emission from the material of the circumstellar disk, this result indicates that the material might not correlate with their ages. Finally, we discovered that one Be candidate, FSR 0904-1, shows longterm variability with an amplitude of $0.2-0.3 \mathrm{mag}$ on the timescale of $\sim 2000$ days. The long-term timescale of the variability implies the timescale of disk evolution might be $\sim 2000$ days.

This work is supported in part by the Ministry of Science and Technology of Taiwan under grants MOST 105-2119-M-008003 (W.-H.I.), MOST 103-2112-M-008-024-MY3 (W.-P.C.), MOST 104-2112-M-008-012-MY3 (C.-C.N.), MOST 1042112-M-008-014-MY3 (C.-K.C), and MOST 106-2917-I-564042 (C.-C.L.). This work is also supported by the Science and Technology Development Fund of Macau (project codes: 039/ 2013/A2 and 017/2014/A1). This publication makes use of data products from the Two Micron All Sky Survey, which is a joint project of the University of Massachusetts and the Infrared Processing and Analysis Center/California Institute of Technology, funded by the National Aeronautics and Space Administration and the National Science Foundation. This publication makes use of data products from the Wide-field Infrared Survey Explorer, which is a joint project of the University of California, Los Angeles, and the Jet Propulsion Laboratory/California Institute of Technology, funded by the National Aeronautics and Space Administration. This research has made use of the WEBDA database, operated at the Department of Theoretical Physics and Astrophysics of the Masaryk University.

\section{Appendix Notes of Individual Clusters}

Basel 10: We identified one Be candidate in this cluster. Kharchenko et al. (2012) used NIR CMD and used the age of turn-off stars to calculate some parameters of this cluster. The distance and age are $2 \mathrm{kpc}$ and $\sim 4 \mathrm{Myr}$, respectively, which are similar to those of Gozha et al. (2012) (1944 pc and $10^{7.6}$ year). Mermilliod et al. (2008) used the spectroscopic method to calculate the mean radial velocity of this cluster and one member of the cluster.

Berkeley 11: We identified one Be candidate in this cluster. Kharchenko et al. (2012) determined that the distance and age are $1.9 \mathrm{kpc}$ and $50 \mathrm{Myr}$, respectively. Dias et al. (2014) identified 79 member stars in this cluster. Leisawitz et al. (1989) conducted a CO survey for 34 open clusters, and they found that molecular clouds are located within/around this cluster.

Dolidze 22: We identified one Be candidate in this cluster. The results of distance and age are $1.7 \mathrm{kpc}$ and $234 \mathrm{Myr}$, respectively (Kharchenko et al. 2012). Dias et al. (2014) have identified 445 member stars in this cluster.

NGC 2244: We identified one Be star candidate in this cluster. This cluster is located in a giant molecular clouds Rosette nebula (Wang et al. 2008). Many Herbig Ae/Be stars are found in this young massive cluster. Banerjee \& Kroupa (2017) used $N$-body simulations to study the cluster size of young massive clusters. They found that the simulated results were much more compact than observed one. There might be some additional mechanisms that affect the expansion of massive clusters.

NGC 869: We identified 14 Be star candidates in this cluster. Mathew \& Subramaniam (2011) used spectroscopic observations to identify 6 Be stars while Marsh Boyer et al. (2012) 
reported $15 \mathrm{Be}$ stars in this cluster. The $\mathrm{V} / \mathrm{R}$ ratio of one of our Be star candidates (NGC 869-26) was found to change from multiple observations during 2006 and 2007, indicating the turbulent nature of the circumstellar disk (Mathew \& Subramaniam 2011). Pan et al. (2016) used BV photometry for an EB-type eclipsing binary and determined the mass ratio was $0.05 \pm 0.001$. The results suggest that the secondary component could be a giant or subgiant star with the outer envelope being stripped.

NGC 884: NGC 884 and NGC 869 could be a binary system of clusters. We identified $12 \mathrm{Be}$ star candidates in this cluster. While Marsh Boyer et al. (2012) identified 13 Be stars in the cluster, Mathew \& Subramaniam (2011) found that one Be star (NGC 884-1) showed spectroscopic variability with a period of 23 months. Using photometric measurements and membership probability, Ahumada \& Lapasset (1995) identified one blue straggler in NGC 884.

NGC 957: We identified one Be star candidate, which is also listed on WEBDA. Schild \& Romanishin (1976) discovered four Be stars while Mathew et al. (2008) have identified two Be stars in NGC 957. Kharchenko et al. (2012) found the distance of NGC 957 to be $1.9 \mathrm{kpc}$, which is similar to that found by Gimenez \& Garcia-Pelayo (1980, $1.85 \mathrm{kpc})$.

Kronberger 12: We identified one $\mathrm{Be}$ candidate in this cluster. Using morphologies, CMD, and stellar density distribution, this cluster was identified as a possible open cluster by Kronberger et al. (2006). Despite a small amount of hot stars, it is one of the two clusters with the highest Be fraction. Our estimation cluster size is about $8^{\prime}$, which is several times larger than the visual size reported form the literature (Dias et al. 2002; Kronberger et al. 2006).

FSR 0749: Two Be candidates that we identified in this old cluster are not catalogued yet in SIMBAD. With a new developed method called the foreground-star-counting technique (Buckner \& Froebrich 2013), the estimated cluster distance and $H$-band extinction are $2.9 \mathrm{kpc}$ and $0.1 \mathrm{mag}$.

NGC 1220: Using $U B V$ photometric observations, Ortolani et al. (2002) argued that NGC 1220 is a young compact cluster with an age of $60 \mathrm{Myr}$ and a distance of $1800+/-200 \mathrm{pc}$. We found the cluster has a radius of 2.2 arcmin, which is similar to that found by Ortolani et al. (2002). Comparing to Kharchenko et al. (2013), they showed that NGC 1220 has an elder age of about $158 \mathrm{Myr}$ and a distance of $2100 \mathrm{pc}$. In this work, we did not find Be star candidates for this cluster. However, Mathew \& Subramaniam (2011) spectroscopically confirmed one Be star with the B5 spectral type in this cluster.

NGC 1348: This is a poorly known open cluster. Carraro (2002) showed that the cluster has a similar distance, $1.9 \mathrm{kpc}$, to that of Kharchenko et al. (2013). Recently, Fang et al. (2012) discovered an active eclipsing binary in the field of NGC 1348. We found no Be star candidates in this cluster.

NGC 1907: NGC 1907 and NGC 1912 are suggested as a binary cluster due to their similar distance (Subramaniam et al. 1995). However, Lee \& Lee (1996) argued that their distance and age difference is so large that they are not physically connected. From orbital motions derived from a Galactic potential model and $N$-body simulations, de Oliveira et al. (2002) suggested that the clusters were born in different regions of the Galaxy and the pair is just a close encounter. No Be stars/candidates were reported in the cluster.

NGC 2269: Kharchenko et al. (2013) obtained a similar distance $(1.58 \mathrm{kpc})$ to that found by Moffat \& Vogt (1975,
$1.44 \mathrm{kpc}$ ). With an age of $457 \mathrm{Myr}$, we reported no Be star candidatess in this cluster.

Berkeley 17: With an age of $(\sim 10 \mathrm{Gyr})$, the cluster is the oldest cluster in our sample. No Be or B-type stars were found in our study of this cluster. It also could be the oldest known open cluster (Salaris et al. 2004). A tail-like structure can be detected with either 2MASS or Pan-STARRS data (Chen et al. 2004; Bhattacharya et al. 2017). Recently, Bhattacharya et al. (2017) discovered more massive than low-mass members in the core, suggesting the cluster should have a mass segregation effect.

FSR 0904: We found one Be star candidate in the cluster. FSR 0904 is a cluster candidate with an age of 6.3 Myr and a distance of $1.43 \mathrm{kpc}$ (Kharchenko et al. 2013). Buckner \& Froebrich (2016) studied 13 FSR clusters and indicated that the cluster should be younger than $80 \mathrm{Myr}$, which is consistent with Kharchenko et al. (2013).

SAI 24: The cluster was identified by Glushkova et al. (2010, see also Collinder 34 in Saurin et al. 2015). The cluster is in a star-forming region associated with Cas OB6. It is a young cluster with an age of $15 \mathrm{Myr}$, and we found no Be star candidates in this cluster.

NGC 744: Three Be candidates were identified as cluster members; two of them are catalogued in SIMBAD known as TYC 3688-1659-1 and TYC 3688-1741-1. Leisawitz et al. (1989) reported four foreground molecular clouds possibly associated with NGC 744.

NGC 743: Our estimation of cluster angular size is nearly $1^{\circ}$ across. This loose cluster had the largest angular size. It harbors the greatest amount of B type stars among billion-year-old clusters. We found four emission-line candidates in which GGA 134 is a known emission-line star (González \& González 1954).

NGC 886: This is one of the oldest clusters in our sample. We identified one Be candidate from the cluster members. It was also reported as an emission-line candidate, VES 734 (Coyne \& MacConnell 1983). The cluster mass within tidal radius is about 400 solar masses (Piskunov et al. 2008).

NGC 1513: We found three cluster members as $\mathrm{Be}$ candidates. They are first reported by del Rio \& Huestamendia (1988) and catalogued in SIMBAD. Two Be candidates, NGC 1513 RH 27 and NGC 1513 RH 47, have a high membership possibility up to $90 \%$ estimated by Frolov et al. (2002).

NGC 1664: The mass of this old cluster is about 1000 solar masses (Piskunov et al. 2008). We identified one Be candidate known as TYC 2906-590-1 in SIMBAD. Recently, van Cauteren et al. (2005) searched variables in this cluster, and two confirmed variable stars with $\delta$ Scuti-like light curves are reported.

NGC 6866: This cluster is in the Cygnus within the Kepler field but there are no Be stars reported in the literature. In 2013, LAMOST obtained 10000 spectra from NGC 6866 (Ren et al. 2016). It will be necessary to further confirm and analyze the photometric and spectroscopic variability of our four Be candidates in this region.

FSR 0253: This cluster not only hosts many Be candidates but has a high Be fraction. We identified $7 \mathrm{Be}$ candidates out of 14 B type stars. In our cluster sample, only two reach such a high Be fraction. However, the other one (Kronberger 12) only has one Be candidate. One Cepheids, VY Cyg, has a probable 
photometric and kinematic relation to FSR 0253 reported by Glushkova et al. (2015).

\section{ORCID iDs}

Po-Chieh Yu (俞伯傑) (10 https://orcid.org/0000-00018894-0854

Chien-De Lee (李建德) (1) https://orcid.org/0000-0002-

3142-7299

I-Chenn Chen (陳以忱) (DD https://orcid.org/0000-00021497-9884

Chow-Choong Ngeow (饒兆聰) (1) https://orcid.org/00000001-8771-7554

Wen-Ping Chen (陳文屏) (1) https://orcid.org/0000-00030262-272X

Jason Surace (iD https://orcid.org/0000-0001-7291-0087

Shrinivas R. Kulkarni (i) https://orcid.org/0000-00015390-8563

\section{References}

Ahumada, J., \& Lapasset, E. 1995, A\&AS, 109, 375

Allen, D. A. 1973, MNRAS, 161, 145

Banerjee, S., \& Kroupa, P. 2017, A\&A, 597, A28

Barbon, R., \& Hassan, S. M. 1973, A\&AS, 10, 1

Barnsley, R. M., \& Steele, I. A. 2013, A\&A, 556, A81

Bessell, M. S., \& Brett, J. M. 1988, PASP, 100, 1134

Bhattacharya, S., Mishra, I., Vaidya, K., \& Chen, W.-P. 2017, ApJ, 847, 138

Bonnell, I. A., \& Davies, M. B. 1998, MNRAS, 295, 691

Buckner, A. S. M., \& Froebrich, D. 2013, MNRAS, 436, 1465

Buckner, A. S. M., \& Froebrich, D. 2016, arXiv:1611.03753

Carciofi, A. C., Okazaki, A. T., Le Bouquin, J.-B., et al. 2009, A\&A, 504, 915 Carraro, G. 2002, A\&A, 387, 479

Chen, L., de Grijs, R., \& Zhao, J. L. 2007, AJ, 134, 1368

Chen, P. S., Liu, J. Y., \& Shan, H. G. 2016, MNRAS, 463, 1162

Chen, W. P., Chen, C. W., \& Shu, C. G. 2004, AJ, 128, 2306

Chojnowski, S. D., Whelan, D. G., Wisniewski, J. P., et al. 2015, AJ, 149, 7 Chojnowski, S. D., Wisniewski, J. P., Whelan, D. G., et al. 2017, AJ, 153, 174

Coyne, G. V., \& MacConnell, D. J. 1983, VatOP, 2, 73

Cui, X.-Q., Zhao, Y.-H., Chu, Y.-Q., et al. 2012, RAA, 12, 1197

Cutri, R. M., Skrutskie, M. F., van Dyk, S., et al. 2003, yCat, 2246, 0

Dachs, J., \& Wamsteker, W. 1982, A\&A, 107, 240

de Grijs, R., Gilmore, G. F., Johnson, R. A., \& Mackey, A. D. 2002, MNRAS, 331,245

del Rio, G., \& Huestamendia, G. 1988, A\&AS, 73, 425

de Oliveira, M. R., Fausti, A., Bica, E., \& Dottori, H. 2002, A\&A, 390, 103

Dias, W. S., Alessi, B. S., Moitinho, A., \& Lépine, J. R. D. 2002, A\&A, 389, 871

Dias, W. S., Monteiro, H., Caetano, T. C., et al. 2014, A\&A, 564, A79

Dougherty, S. M., Taylor, A. R., \& Clark, T. A. 1991, AJ, 102, 1753

Drew, J. E., Greimel, R., Irwin, M. J., et al. 2005, MNRAS, 362, 753

Fabregat, J., \& Torrejón, J. M. 2000, A\&A, 357, 451

Fang, X.-S., Gu, S.-H., Hui, H.-K., et al. 2012, RAA, 12, 93

Frolov, V. N., Jilinski, E. G., Ananjevskaja, J. K., et al. 2002, A\&A, 396, 125

Gimenez, A., \& Garcia-Pelayo, J. 1980, A\&AS, 41, 9

Girardi, L., Bertelli, G., Bressan, A., et al. 2002, A\&A, 391, 195

Glushkova, E. V., Koposov, S. E., Zolotukhin, I. Y., et al. 2010, AstL, 36, 75

Glushkova, E. V., Zabolotskikh, M. V., Rastorguev, A. S., Grudskaya, A. V., \& Koposov, S. E. 2015, BaltA, 24, 360

González, G., \& González, G. 1954, BOTT, 1, 3

Gozha, M. L., Koval', V. V., \& Marsakov, V. A. 2012, AstL, 38, 519

Haubois, X., Carciofi, A. C., Rivinius, T., Okazaki, A. T., \& Bjorkman, J. E. 2012, ApJ, 756, 156

Hsia, C.-H., \& Zhang, Y. 2014, A\&A, 563, A63

Hubert, A. M., \& Floquet, M. 1998, A\&A, 335, 565

Hubert, A. M., Floquet, M., Hao, J. X., et al. 1997, A\&A, 324, 929

Jaschek, M., Slettebak, A., \& Jaschek, C. 1981, Be star terminology., Be Star Newsletter, 4, 9

Keller, S. C., Grebel, E. K., Miller, G. J., \& Yoss, K. M. 2001, AJ, 122, 248

Kharchenko, N. V., Piskunov, A. E., Schilbach, E., Röser, S., \& Scholz, R.-D. 2012, A\&A, 543, A156
Kharchenko, N. V., Piskunov, A. E., Schilbach, E., Röser, S., \& Scholz, R.-D. 2013, A\&A, 558, A53

Klement, R., Carciofi, A. C., Rivinius, T., et al. 2015, A\&A, 584, A85

Kourniotis, M., Bonanos, A. Z., Soszyński, I., et al. 2014, A\&A, 562, A125

Kraus, S., Monnier, J. D., Che, X., et al. 2012, ApJ, 744, 19

Kronberger, M., Teutsch, P., Alessi, B., et al. 2006, A\&A, 447, 921

Kubát, J., Korčáková, D., Kawka, A., et al. 2007, A\&A, 472, 163

Kulkarni, S. R. 2013, ATel, 4807, 1

Kulkarni, S. R. 2016, in AAS Meeting 227 Abstracts, 314.01

Labadie-Bartz, J., Pepper, J., McSwain, M. V., et al. 2017, AJ, 153, 252

Laher, R. R., Surace, J., Grillmair, C. J., et al. 2014, PASP, 126, 674

Law, N. M., Kulkarni, S. R., Dekany, R. G., et al. 2009, PASP, 121, 1395

Lee, C.-D., \& Chen, W.-P. 2011, in IAU Symp. 272, Active OB Stars: Structure, Evolution, Mass Loss, and Critical Limits, ed. C. Neiner et al. (Cambridge: Cambridge Univ. Press), 366

Lee, C.-D., Liu, S.-Y., \& Chen, W.-P. 2016, in ASP Conf. Ser. 506, Bright Emissaries: Be Stars as Messengers of Star-Disk Physics, ed. T. A. A. Sigut \& C. E. Jones (San Francisco, CA: ASP), 111

Lee, S. H., \& Lee, S.-W. 1996, PKAS, 11, 139

Lee, U., Osaki, Y., \& Saio, H. 1991, MNRAS, 250, 432

Leisawitz, D., Bash, F. N., \& Thaddeus, P. 1989, ApJS, 70, 731

Lin, C.-C., Hou, J.-L., Chen, L., et al. 2015, RAA, 15, 1325

Littlefair, S. P., Naylor, T., Jeffries, R. D., Devey, C. R., \& Vine, S. 2003, MNRAS, 345, 1205

Majewski, S. R., Schiavon, R. P., Frinchaboy, P. M., et al. 2015, arXiv:1509. 05420

Marsh Boyer, A. N., McSwain, M. V., Aragona, C., \& Ou-Yang, B. 2012, AJ, 144,158

Martayan, C., Baade, D., \& Fabregat, J. 2009, in IAU Symp. 256, The Magellanic System: Stars, Gas, and Galaxies, ed. J. T. Van Loon \& J. M. Oliveira (Cambridge: Cambridge Univ. Press), 349

Mathew, B., \& Subramaniam, A. 2011, BASI, 39, 517

Mathew, B., Subramaniam, A., \& Bhatt, B. C. 2008, MNRAS, 388, 1879

McNally, D. 1965, Obs, 85, 166

McSwain, M. V., \& Gies, D. R. 2005, ApJS, 161, 118

McSwain, M. V., Huang, W., \& Gies, D. R. 2009, ApJ, 700, 1216

Mennickent, R. E., Pietrzyński, G., Gieren, W., \& Szewczyk, O. 2002, A\&A, 393, 887

Mermilliod, J. C., Mayor, M., \& Udry, S. 2008, A\&A, 485, 303

Merrill, P. W., Humason, M. L., \& Burwell, C. G. 1925, ApJ, 61, 389

Moffat, A. F. J., \& Vogt, N. 1975, A\&AS, 20, 85

Morgan, W. W., Code, A. D., \& Whitford, A. E. 1955, ApJS, 2, 41

Okazaki, A. T. 1997, A\&A, 318, 548

Ortolani, S., Carraro, G., Covino, S., Bica, E., \& Barbuy, B. 2002, A\&A, 391,179

Pan, Y., Luo, Z.-Q., Zhang, X.-B., et al. 2016, RAA, 16, 109

Pápics, P. I., Tkachenko, A., Van Reeth, T., et al. 2017, A\&A, 598, A74

Paul, K. T., Subramaniam, A., Mathew, B., \& Shruthi, S. B. 2017, NewA, 56,28

Paunzen, E., Schnell, A., \& Maitzen, H. M. 2005, A\&A, 444, 941

Pecaut, M. J., \& Mamajek, E. E. 2013, ApJS, 208, 9

Pecaut, M. J., Mamajek, E. E., \& Bubar, E. J. 2012, ApJ, 746, 154

Percy, J. R., Hosick, J., Kincaide, H., \& Pang, C. 2002, PASP, 114, 551

Piskunov, A. E., Schilbach, E., Kharchenko, N. V., Röser, S., \& Scholz, R.-D. 2008, A\&A, 477, 165

Pojmanski, G. 1997, AcA, 47, 467

Pojmański, G. 2001, in ASP Conf. Ser. 246, IAU Coll. 183: Small Telescope Astronomy on Global Scales, ed. B. Paczynski, W.-P. Chen, \& C. Lemme (San Francisco, CA: ASP), 53

Porter, J. M., \& Rivinius, T. 2003, PASP, 115, 1153

Raboud, D., \& Mermilliod, J.-C. 1998, A\&A, 333, 897

Raddi, R., Drew, J. E., Steeghs, D., et al. 2015, MNRAS, 446, 274

Ren, A., Fu, J., De Cat, P., et al. 2016, ApJS, 225, 28

Rieke, G. H., \& Lebofsky, M. J. 1985, ApJ, 288, 618

Rivinius, T., Baade, D., \& Carciofi, A. C. 2016, A\&A, 593, A106

Rivinius, T., Baade, D., \& Stefl, S. 2003, A\&A, 411, 229

Rivinius, T., Carciofi, A. C., \& Martayan, C. 2013, A\&ARv, 21, 69

Roeser, S., Demleitner, M., \& Schilbach, E. 2010, AJ, 139, 2440

Sabogal, B. E., García-Varela, A., \& Mennickent, R. E. 2014, PASP, 126, 219

Saio, H., Ekström, S., Mowlavi, N., et al. 2017, MNRAS, 467, 3864

Salaris, M., Weiss, A., \& Percival, S. M. 2004, A\&A, 414, 163

Saurin, T. A., Bica, E., \& Bonatto, C. 2015, MNRAS, 448, 1687

Schild, R., \& Romanishin, W. 1976, ApJ, 204, 493

Souza, S. P., Garcia Soto, A., \& Wong, H. 2016, in AAS Meeting 228 Abstracts, 319.03

Subramaniam, A., Gorti, U., Sagar, R., \& Bhatt, H. C. 1995, A\&A, 302, 86 
Tarasov, A. E., \& Malchenko, S. L. 2012, AstL, 38, 428

Townsend, R. H. D., Owocki, S. P., \& Howarth, I. D. 2004, MNRAS, 350, 189 van Cauteren, P., Lampens, P., Robertson, C. W., \& Strigachev, A. 2005, CoAst, 146,21

Wang, J., Townsley, L. K., Feigelson, E. D., et al. 2008, ApJ, 675, 464

Wisniewski, J. P., \& Bjorkman, K. S. 2006, ApJ, 652, 458

Wisniewski, J. P., Bjorkman, K. S., Magalhães, A. M., et al. 2007, ApJ, 671, 2040
Woolf, N. J., Stein, W. A., \& Strittmatter, P. A. 1970, A\&A, 9, 252

Wright, E. L., Eisenhardt, P. R. M., Mainzer, A. K., et al. 2010, AJ, 140, 1868

Yu, P. C., Lin, C. C., Chen, W. P., et al. 2015, AJ, 149, 43

Yu, P.-C., Lin, C.-C., Lin, H.-W., et al. 2016, AJ, 151, 121

Zhang, C., Fu, J. N., \& Jiang, X. J. 2008, JPhCS, 118, 012078

Zhang, P., Chen, P. S., \& Yang, H. T. 2005, NewA, 10, 325 